\title{
Modeling of slow time-scale behavior of fast molecular dynamic systems
}

\author{
Likun Tan*, Amit Acharya, Kaushik Dayal \\ Civil and Environmental Engineering, Carnegie Mellon University
}

\begin{abstract}
This paper aims to apply a recently developed numerical scheme towards multi-time scale modeling, which we refer to as 'Practical Time Averaging' (PTA), to molecular dynamical (MD) systems. In the first part, we investigate the fine-scale dynamics of a one-dimensional chain of identical particles under cyclic loading. Assuming a double-well interatomic potential among adjacent particles leads to a phase transition between two distinct equilibrium states. Specifically, we study the macroscopic stress-strain behavior of the dynamical chain in three settings, i.e. Newtonian MD, Newtonian MD with viscous dissipation and Newtonian MD with thermostat. Rate-independent high frequency oscillations are observed in Newtonian MD due to an instability that is related to the non-convexity of the strain energy. This is stabilized by adding viscosity or a thermostat, which leads to strong hysteresis that is consistent with quasi-static results (i.e. lattice statics). In the second part, we first define coarse variables as finite time averages of phase functions in MD. Then we apply the technique of PTA developed in Tan, Acharya and Dayal [16] to numerically approximate the coarse dynamics for the time averaged quantities. The tested model problems include a two-dimensional lattice made of stoichiometric Nickel-Manganese undergoing detwinning and a three-dimensional atomic chain made of face-centered cubic (FCC) Nickel under uniaxial tension. The macroscopic features (such as space-time averaged strain/stress) are obtained from coarse dynamics. It is also shown that the time savings become significant when the loading rate is small.
\end{abstract}

Keywords: Molecular Dynamics; Phase transition; Time-averaging; Multi-scale modeling

\section{Introduction}

Molecular dynamics (MD) has become an important simulation tool in understanding material behaviors in a vast variety of settings. This method is based on a particle description of atoms or molecules and treats the interaction between the particles using Newtonian dynamics. However, a key difficulty in using MD for engineering applications is the extremely large separation between the timescales of atomic bond vibrations (femtoseconds) and even the smallest timescales of engineering interest (nano to micro seconds). This limits MD to unrealistically small time intervals for many applications. This situation is even worse for materials under extremely slow loading rates. When the loading rate tends to zero, the equations of motion become a singularly perturbed system of Ordinary Differential Equations (ODE). The objective of this paper is to apply and evaluate the temporal coarse-graining strategy developed in [16] to such singularly perturbed problems in the mechanics of materials.

In the first part of the paper, we study the macroscopic stress-strain behavior of a onedimensional dynamical chain of atoms joined by springs with a non-monotone stress-strain relation. A sinusoidal loading with small loading rate is applied at one end of the chain. The magnitude of the external loading is large enough so that the chain will go from one equilibrium state to the other during this dynamical process. Due to the non-monotonicity in the constitutive relation (or equivalently, the non-convex interatomic potential), the dynamics will lead to rate-independent high frequency vibration of the atoms beyond a certain critical strain as a result of an instability of the system (reported before in [7] and called the 'inner

\footnotetext{
${ }^{*}$ Tel.: +1 4123308044 .

E-mail address: likunt@caltech.edu
} 
instability') that is insensitive to external excitation. This finding is quite different from the observation under quasi-static dynamics that ignores inertia, which is normally associated with a hysteresis indicating energy dissipation at the macroscopic level. It is also suggested in [7] that the inner vibrations of the atoms can be stabilized by a small dissipation, which leads to a strong hysteresis rather than a steady state stress-strain dependence. We test two cases in order to dissipate the high kinetic energy of the system. One is by adding viscosity and the other is by thermostatting. The effects in both cases are essentially the same and thus give rise to quite similar results. Furthermore, the hysteresis converges to the quasi-static result as the loading rate tends to zero.

In the second part of the paper, a numerical strategy developed in [16] and referred to as 'Practical Time Averaging'(PTA) is applied to two MD problems. The approach may be viewed as a natural extension of the method of averaging motivated by practical considerations, naturally for systems whose limiting fast flows may not converge to an equilibrium. The theory of $[5,4]$ is utilized here, where the limit behavior of the fast flow (parameterized by the slow variable) in a singulary perturbed system is characterized by an invariant measure rather than algebraic equations in the standard reduced order approach. However, this theory requires that variables that can be averaged be 'slow', i.e. orthogonal to the fast flow in a precise sense, but does not provide a prescription for generating such 'orthogonal observables'. Following [15] (which makes rigorous some of the ideas presented in [2,3]) and [16], a natural class of slow variables based on time averaged coarse observables and their evolution are defined and practically implemented.

Two model problems are investigated with the implementation of PTA. One is a twodimensional infinite strip of a Ni-Mn lattice with two variants of martensite coexisting in the lattice. A twin boundary is located within the strip in a stress-free initial configuration. The two variants have the same free energy. When the body is subjected to an applied cyclic loading, the free-energies of the different variants are no longer equal and one variant becomes energetically more favorable than the other. This leads to the motion of the twin boundary and the dynamics of detwinning. We study the macroscopic stress-strain behavior of the two-dimensional lattice. The other tested example is a three-dimensional dynamical chain system made of FCC Ni undergoing uniaxial tension. The macroscopic stress-strain behavior with different loading rates is studied. We define the overall stress and strain as coarse variables. The averaged response of fine-scale dynamics and PTA are found to be in broad agreement. Moreover, in the range of slow loading rate, we show that the time savings from PTA is significant in both examples.

This paper is organized as follows: In Section 2, we construct a one-dimensional atomic dynamical chain under cyclic loading and study the stress-strain behavior in three different settings. Section 3 is excerpted from [16], which shows the procedures of PTA to write down the coarse evolution equation for singularly perturbed systems based on the Young measure theory (i.e. $[5,4,15,16]$ ). In Section 4 and 5 , we implement the idea of PTA on a two-dimensional $\mathrm{Ni}-\mathrm{Mn}$ system and a three-dimensional atomic chain made of FCC Ni, respectively. The stressstrain relations from fine dynamics and from PTA are investigated in both cases. We end in Section 6 with summarizing the main findings from the various numerical tests and discussing the advantages as well as the limitations of the proposed multi-scale modeling technique.

\section{Atomistic Study}

\subsection{MODEL DESCRIPTION}

The dynamics of interest is adopted from [14]. This problem is chosen since the set-up is quite similar to large scale MD.

Figure 1 shows a chain of atoms interacting with each other through non-linear springs with two states of equilibrium. The relation between the potential energy and the distances of atoms 
is also plotted in Figure 1. The interactions are limited to the first nearest neighbors.

In this example, we assume double-well potential energy, i.e.,

$$
\phi=\sum_{i=1}^{N-1} \phi^{1}\left(x_{i+1}-x_{i}\right)=\sum_{i=1}^{N-1}\left(x_{i+1}-x_{i}-a_{s}\right)^{2}\left(x_{i+1}-x_{i}-a_{l}\right)^{2},
$$

where $x_{i}$ denotes the position of mass $i$ at any time. $a_{s}$ is the equilibrium separation between two adjacent masses in the low strain phase and $a_{l}$ is the equilibrium separation between them in the high strain phase. The initial configuration of the atoms is chosen to be in the low strain phase, so the displacement of atom $i$ can be written as

$$
u_{i}=x_{i}-(i-1) a_{s} .
$$

The potential energy becomes

$$
\phi=\sum_{i=1}^{N-1} \phi^{1}\left(u_{i+1}-u_{i}\right)=\sum_{i=1}^{N-1}\left(u_{i+1}-u_{i}\right)^{2}\left(u_{i+1}-u_{i}-\left(a_{l}-a_{s}\right)\right)^{2} .
$$

We consider a dynamical system with the first atom fixed at the left end and a sinusoidal force applied at the right end. Let the applied force be

$$
L(t)=A \sin (\omega t) .
$$

By introducing another variable $g(t)$ to denote the time derivative of $L(t)$, we have the autonomous system

$$
\begin{aligned}
& \dot{L}=\omega g, \\
& \dot{g}=-\omega L .
\end{aligned}
$$

We assume the mass of each atom to be $m_{i}=1 \forall i$ and $u_{1}=v_{1}=0$. The fine theory can be written as

$$
\begin{aligned}
& \dot{u}_{i}=v_{i}, \quad \forall i \text { from } 2 \text { to } N, \\
& \dot{v}_{i}=-\frac{\partial \phi}{\partial u_{i}}, \quad \forall i \text { from } 2 \text { to } N-1, \\
& \dot{v}_{N}=-\frac{\partial \phi}{\partial u_{N}}+L, \\
& \dot{L}=\omega g, \quad \dot{g}=-\omega L .
\end{aligned}
$$
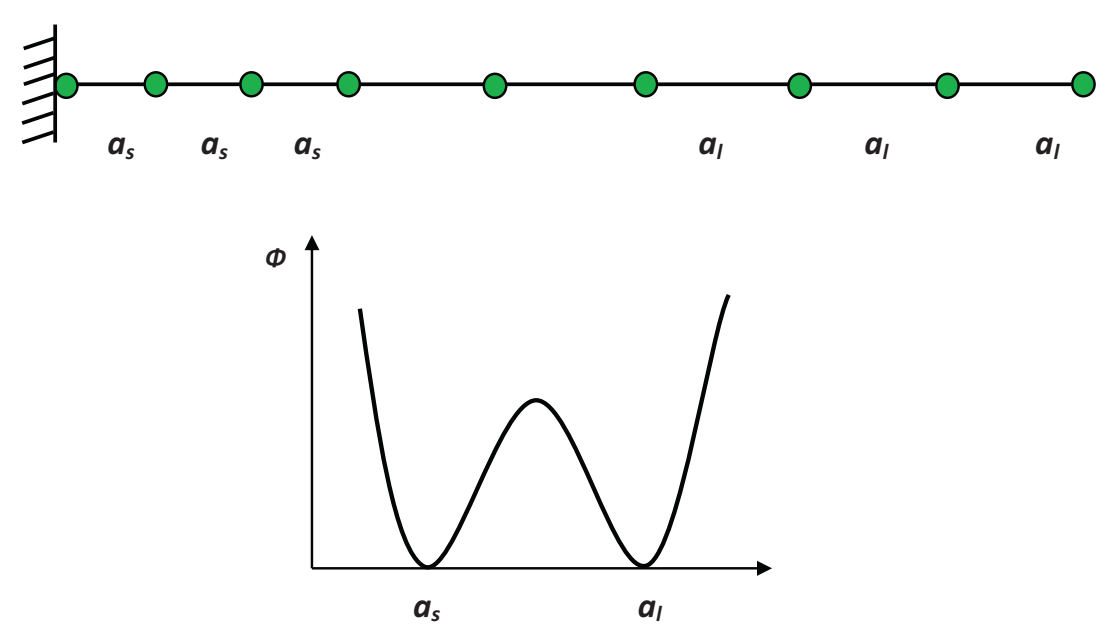

Figure 1: One-dimensional chain of interacting masses. The graph below shows the interaction potentials for the connecting nonlinear springs. 
The partial derivatives in the above equations are dependent on the displacement of atoms, denoted as

$$
\begin{aligned}
\frac{\partial \phi}{\partial u_{i}} & =\Lambda_{1}+\Lambda_{2}, \quad \forall i \text { from } 2 \text { to } N-1, \\
\frac{\partial \phi}{\partial u_{N}} & =\Lambda_{2}
\end{aligned}
$$

where

$$
\begin{aligned}
& \Lambda_{1}=-2\left(u_{i+1}-u_{i}\right)\left(u_{i+1}-u_{i}-\left(a_{l}-a_{s}\right)\right)\left(2\left(u_{i+1}-u_{i}\right)-\left(a_{l}-a_{s}\right)\right), \\
& \Lambda_{2}=2\left(u_{i}-u_{i-1}\right)\left(u_{i}-u_{i-1}-\left(a_{l}-a_{s}\right)\right)\left(2\left(u_{i}-u_{i-1}\right)-\left(a_{l}-a_{s}\right)\right) .
\end{aligned}
$$

\subsection{HAMILTONIAN VS. DISSIPATION}

As is to be expected and shown in [7] (as well as from our numerical study), in Newtonian MD there is no heat loss during the cyclic loading at the microscopic level (it also shows no hysteresis even at the macroscopic level, as evidenced by averaged stress-strain curves in our numerical tests). In [7], it is suggested that hysteresis can be obtained by adding some viscosity to the system, therefore we test the cases of Newtonian MD, damped system and MD with thermostat. In all the cases, we consider both load control and strain control devices. The strain-load curves are demonstrated in each case.

\subsubsection{Newtonian MD}

Newtonian MD consists of an isolated system with no heat transfer with the outside. The energy is conserved in the sense that the external work is transferred to the total increase of the potential energy and the kinetic energy of the system. The governing equations of this type of system follow Hamiltonian dynamics.

\section{a. Load Control}

The equations of motion in the load control case are exactly (6).

\section{b. Strain Control}

In the case of strain control, the load is applied to the system by controlling the displacement of the atom at the free end, which is defined as

$$
u_{N}=\left(\frac{A_{2}-A_{1}}{2}\right) \sin \left(\omega t-\theta_{0}\right)+\frac{A_{1}+A_{2}}{2},
$$

where $A_{1}$ and $A_{2}$ are the lowest and highest amplitudes of the displacement of the last atom, respectively. We set them to be

$$
A_{1}=-(N-1) \cdot A_{0}, \quad A_{2}=(N-1) \cdot\left(a_{l}-a_{s}\right)+(N-1) \cdot A_{0},
$$

such that the averaged strain will go from $-A_{0}$ to $\left(a_{l}-a_{s}\right)+A_{0}$, where $A_{0}$ is an arbitrary constant (usually we want $A_{0}$ to be significantly smaller than $a_{l}-a_{s}$ ). $\theta_{0}$ is the phase shift, which is determined by

$$
\theta_{0}=\arcsin \left(\frac{A_{1}+A_{2}}{A_{2}-A_{1}}\right)
$$


so as to satisfy $u_{N}(0)=0$. With these definitions in hand, the equations of motion are

$$
\begin{aligned}
& \dot{u}_{i}=v_{i}, \quad \forall i \text { from } 2 \text { to } N-1, \\
& \dot{v}_{i}=-\frac{\partial \phi}{\partial u_{i}}, \quad \forall i \text { from } 2 \text { to } N-1, \\
& \text { with } \quad u_{N}=\left(\frac{A_{2}-A_{1}}{2}\right) \sin \left(\omega t-\theta_{0}\right)+\frac{A_{1}+A_{2}}{2} .
\end{aligned}
$$

From Newton's second law, the external load is

$$
L=\frac{\partial \phi}{\partial u_{N}}+\ddot{u}_{N} .
$$

\subsubsection{Damped System}

a. Load Control

For the case where damping is added to the system, the equations of motion become

$$
\begin{aligned}
& \dot{u}_{i}=v_{i}, \quad \forall i \text { from } 2 \text { to } N, \\
& \dot{v}_{i}=-c v_{i}-\frac{\partial \phi}{\partial u_{i}}, \quad \forall i \text { from } 2 \text { to } N-1, \\
& \dot{v}_{N}=-c v_{N}-\frac{\partial \phi}{\partial u_{N}}+L, \\
& \dot{L}=\omega g, \quad \dot{g}=-\omega L,
\end{aligned}
$$

where $c$ is the damping coefficient.

b. Strain Control

In the strain control case, we have

$$
\begin{aligned}
& \dot{u}_{i}=v_{i}, \quad \forall i \text { from } 2 \text { to } N-1, \\
& \dot{v}_{i}=-c v_{i}-\frac{\partial \phi}{\partial u_{i}}, \quad \forall i \text { from } 2 \text { to } N-1, \\
& \text { with } \quad u_{N}=\left(\frac{A_{2}-A_{1}}{2}\right) \sin \left(\omega t-\theta_{0}\right)+\frac{A_{1}+A_{2}}{2} .
\end{aligned}
$$

The loading on the last atom is given by

$$
L=\frac{\partial \phi}{\partial u_{N}}+c v_{N}+\ddot{u}_{N} .
$$

\subsubsection{MD with Thermostat}

We consider the dynamical system of the atomic chain at a constant temperature by thermostatting. The primary purpose is to control the temperature of the system, thus to control the total kinetic energy of atoms. As observed in [7], a chain with non-convex interaction potential energy between atoms will go through high frequency oscillations, indicating an unstable dynamical process due to the negative stiffness in the constitutive relation beyond certain critical strain. 
This is the inner instability of the system in the sense that the intensity of the vibrations is determined by the structure of the system, and is independent of the rate of the external loading. Our aim is to remove the inner vibrations of atoms by keeping the temperature of the system at a low value, with an expectation to see hysteresis in the macroscopic stress-strain behavior. A good review on thermostat algorithms based on Newtonian MD scheme can be found in [10]. We choose the Woodcock algorithm [17] in the context of the leap-frog integrator to maintain a constant temperature. In this algorithm, a scaling factor $\lambda$ is introduced and determined by constraining the instantaneous temperature $\mathcal{T}$ to be equal to a reference temperature $\mathcal{T}_{0}$ at each time step. In the following, we describe the algorithm for both load control and strain control cases.

\section{a. Load Control}

The equations of motion are given as

$$
\begin{aligned}
& \ddot{u}_{i}=-c(t) \dot{u}_{i}-\frac{\partial \phi}{\partial u_{i}}, \quad \forall i \text { from } 2 \text { to } N-1, \\
& \ddot{u}_{N}=-c(t) \dot{u}_{N}-\frac{\partial \phi}{\partial u_{N}}+L .
\end{aligned}
$$

Note here that $c(t)$ is like a control parameter. When the temperature of the system is higher than the reference temperature $\mathcal{T}_{0}, c$ is positive, which means the system will transfer heat into the environment. On the other hand, $c$ becomes negative when the temperature is lower than the reference temperature, indicating that the system will absorb heat from the environment.

We apply the Woodcock algorithm $[17,10]$ to compute the scaling factor $\lambda$ that is directly used to modify the leap-frog algorithm in the case of thermostat. The instantaneous temperature is defined as

$$
\mathcal{T}=\left(\frac{1}{2 N} \sum_{i=1}^{N}\left(v_{i}-\left\langle v_{i}\right\rangle\right)^{2}\right)^{1 / 2}=\left(\frac{1}{2}\left(\left\langle v_{i}^{2}\right\rangle-\left\langle v_{i}\right\rangle^{2}\right)\right)^{1 / 2},
$$

where

$$
\langle\cdot\rangle=\frac{1}{N} \sum_{i=1}^{N}(\cdot) .
$$

In classical MD, the leap-frog algorithm is presented as

$$
\begin{aligned}
& u_{i}(t)=u_{i}(t-\triangle t)+v_{i}\left(t-\frac{\triangle t}{2}\right) \triangle t, \\
& v_{i}\left(t+\frac{\triangle t}{2}\right)=v_{i}\left(t-\frac{\triangle t}{2}\right)+\ddot{u}_{i}(t) \triangle t .
\end{aligned}
$$

For thermostat to be imposed on the system, the scaling factor $\lambda$ is computed as follows,

$$
\begin{aligned}
& u_{i}(t)=u_{i}(t-\triangle t)+v_{i}\left(t-\frac{\triangle t}{2}\right) \triangle t, \\
& v_{i}\left(t+\frac{\triangle t}{2}\right)=\lambda(t ; \triangle t) v_{i}^{\prime}\left(t+\frac{\triangle t}{2}\right)=\lambda(t ; \triangle t)\left[v_{i}\left(t-\frac{\triangle t}{2}\right)+\ddot{u}_{i}(t) \triangle t\right],
\end{aligned}
$$

where $v_{i}^{\prime}\left(t+\frac{\Delta t}{2}\right)$ is approximated from classical Newton's equations without damping.

Imposing the constrain $\lambda(t ; 0)=1$, we can show that

$$
c(t)=-\lim _{\triangle t \rightarrow 0} \frac{\lambda(t ; \triangle t)-1}{\triangle t}=-\left.\frac{\partial \lambda(t ; \triangle t)}{\partial \triangle t}\right|_{\triangle t=0} .
$$


From the definition of temperature, we have

$$
\mathcal{T}\left(t+\frac{\triangle t}{2}\right)=\left[\frac{1}{2}\left\langle\left(v_{i}\left(t+\frac{\triangle t}{2}\right)\right)^{2}\right\rangle-\frac{1}{2}\left\langle v_{i}\left(t+\frac{\triangle t}{2}\right)\right\rangle^{2}\right]^{1 / 2}=\mathcal{T}_{0} .
$$

Substitute the second equation of (21) into (23),

$$
\begin{aligned}
& \left(\mathcal{T}\left(t+\frac{\triangle t}{2}\right)\right)^{2}=\frac{\lambda^{2}}{2}\left\langle\left(v_{i}\left(t-\frac{\triangle t}{2}\right)+\ddot{u}_{i}(t) \triangle t\right)^{2}\right\rangle \\
& -\frac{\lambda^{2}}{2}\left\langle v_{i}\left(t-\frac{\triangle t}{2}\right)+\ddot{u}_{i}(t) \triangle t\right\rangle^{2}=\mathcal{T}_{0}^{2} .
\end{aligned}
$$

Denote

$$
v_{i}=v_{i}\left(t-\frac{\triangle t}{2}\right), \quad u_{i}=u_{i}(t)
$$

for simplicity. From (24), we can solve for $\lambda$, that is,

$$
\lambda=\frac{\mathcal{T}_{0}}{\left[\frac{1}{2}\left\langle\left(v_{i}+\ddot{u}_{i} \triangle t\right)^{2}\right\rangle-\frac{1}{2}\left\langle v_{i}+\ddot{u}_{i} \triangle t\right\rangle^{2}\right]^{1 / 2}}=\frac{\mathcal{T}_{0}}{\mathcal{T}^{\prime}\left(t+\frac{\Delta t}{2}\right)},
$$

where $\mathcal{T}^{\prime}\left(t+\frac{\Delta t}{2}\right)$ denotes the instantaneous temperature at the time $t+\frac{\Delta t}{2}$ from Newton's equation.

We can check that

$$
\lambda(t ; 0)=\frac{\mathcal{T}_{0}}{\left[\frac{1}{2}\left\langle v_{i}^{2}\right\rangle-\frac{1}{2}\left\langle v_{i}\right\rangle^{2}\right]^{1 / 2}}=1 .
$$

Thus the control parameter $c$ can be determined following (22). Note that even though this model is physically unrealistic (a 'structural' ODE system with negative damping), the algorithm is stable as long as the denominator in (26) is nonzero regardless of the sign of $c$.

b. Strain Control

The equations of motion are

$$
\begin{aligned}
& \ddot{u}_{i}=-c(t) \dot{u}_{i}-\frac{\partial \phi}{\partial u_{i}}, \quad \forall i \text { from } 2 \text { to } N-1, \\
& \text { with } u_{N}=\left(\frac{A_{2}-A_{1}}{2}\right) \sin \left(\omega t-\theta_{0}\right)+\frac{A_{1}+A_{2}}{2} .
\end{aligned}
$$

The velocity of the last atom becomes

$$
v_{N}=\omega\left(\frac{A_{2}-A_{1}}{2}\right) \cos \left(\omega t-\theta_{0}\right),
$$

and can be assumed to be zero for slow loadings. The procedures for computing the coefficient $\lambda$ and the control parameter $c$ are the same as before, except we can take $v_{N}=0$.

The loading on the last atom is again determined by

$$
L=\frac{\partial \phi}{\partial u_{N}}+c(t) v_{N}+\ddot{u}_{N}
$$




\subsection{NUMERICAL EXPERIMENTS}

The next series of computer simulations aims to study the load-strain response of the atomic chain under a slow external force, with the strain defined as

$$
\varepsilon=\frac{1}{N-1} \sum_{i=2}^{N}\left(u_{i}-u_{i-1}\right)=\frac{u_{N}}{N-1} .
$$

The number of atoms is set to be 2000. Our numerical observation shows that in both the load control and strain control cases (see Figure 2(a) and 3(a), respectively), the atomic chain goes through high frequency oscillations when the strain between any two adjacent atoms becomes greater than the critical strain (thus the stiffness in the constitutive relation becomes negative). In our case, the critical strain equals $(1 / 2-\sqrt{3} / 6)\left(a_{l}-a_{s}\right)$. This dynamical problem has been studied by Balk et al [7]. Here we excerpt some main findings from their work:

1. "When the elongation of one of the springs becomes greater than the critical elongation, the springs start to oscillate with "high" frequencies, no matter how slowly we increase the total length of the chain. This is another reason to say that this mass spring structure is unstable: high frequency oscillations appear in the structure while stress changes slowly".

2. "In unstable chains, the inner instabilities excite an intense dynamical process. This process is determined by the structure of the system. Its intensity is not small independently of the rate of the external elongation".

3. "The inner vibrations can be stabilized by a small dissipation. The introduced dissipation leads to a strong hysteresis instead of a steady state stress-strain dependence".

In Newtonian MD, the energy is conserved 'microscopically' in the sense that the applied work has been totally transferred to the mechanical energy (i.e. potential energy plus high atomic kinetic energy), thus no hysteresis at the microscopic level. It is generally believed that this process is irreversible and the high atomic oscillation is considered as heat loss at the macroscopic level. However, we show the absence of hysteresis in the 'macroscopic' response (i.e. time-averaged load-strain behavior defined below), as can be seen in Figure 4, where the time-averaged variable is defined as

$$
\overline{(\cdot)}(t):=\frac{1}{\tau} \int_{t}^{t+\tau}(\cdot)(s) d s .
$$

In this calculation, we set $\tau$ to be around 400 of the natural period of atomic vibration.

Based on the last finding pointed out in [7], we construct damped systems with different damping coefficients $c$. The strain vs. load curves are plotted in Figure 2(b) and 3(b), corresponding to the load control and strain control cases, respectively. The natural frequency $\omega_{0}$ of the oscillation of two neighboring masses is about 10, so the damping coefficients are considerably smaller than this value. We observe strong hysteresis in the cases of $c=1 \mathrm{e}-2, c=0.05$ and $c=0.1$, while the hysteresis is not so obvious in the case of $c=1 \mathrm{e}-4$.

Figure 5 and 6 describe the strain vs. load relations of the system with thermostat, under load control and strain control, respectively. We set the number of atoms to be 1000 . In all the tests, the temperature of the system is a constant equal to the reference temperature $\mathcal{T}_{0}$. We find that by maintaining the instantaneous temperature at a low value (for example, $\mathcal{T}_{0}=1$ ), the hysteresis is obvious, as shown in Figure 5(a) and 6(a). However, when setting a high temperature to the system (for example, $\mathcal{T}_{0}=10$ ), the hysteresis is hardly observed. The physical explanation of this phenomenon is the following: The kinetic energy is allowed to be 
(a)

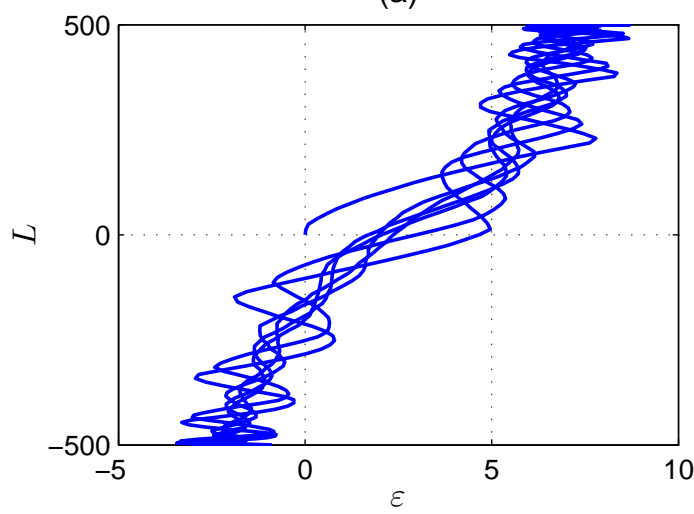

(b)

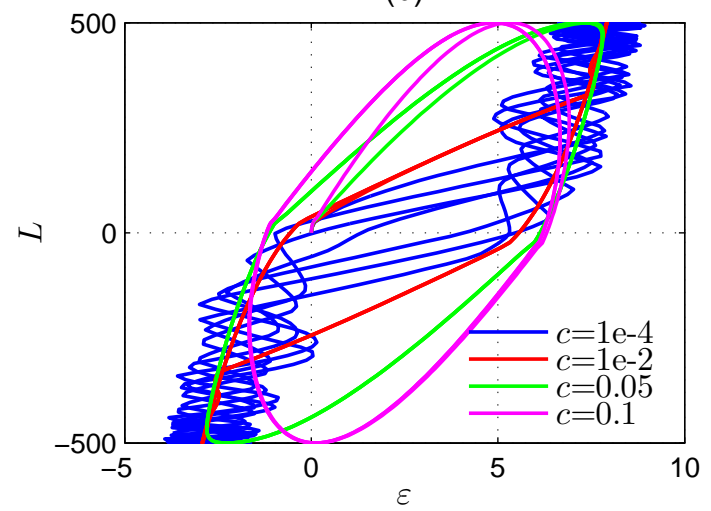

Figure 2: (a) Newtonian MD under load control; (b) Damped system under load control

(a)

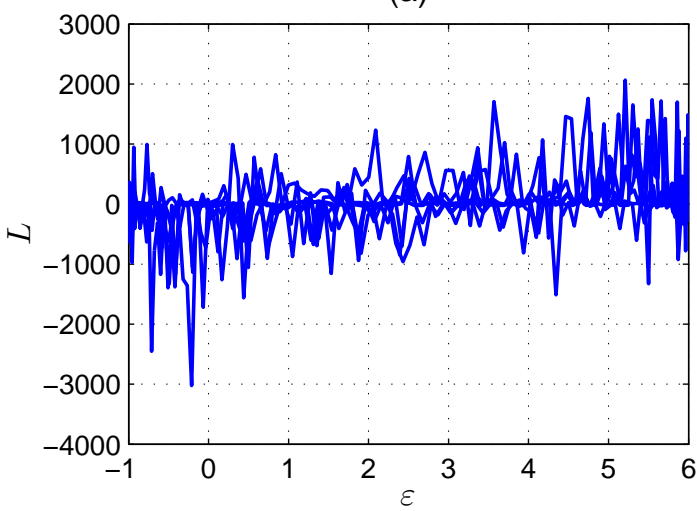

(b)

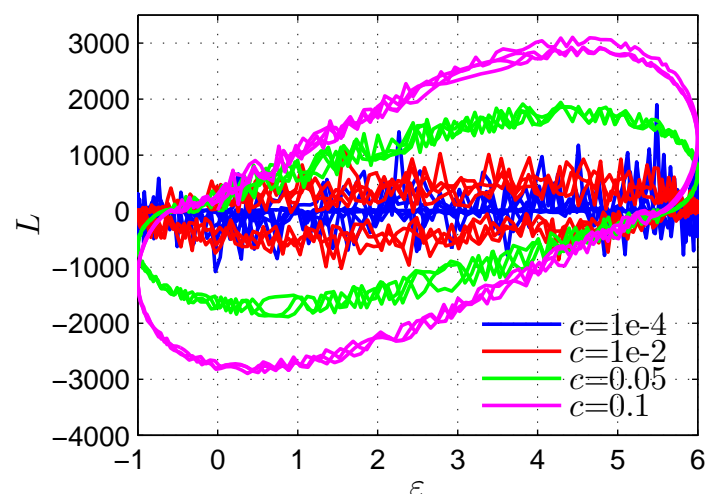

Figure 3: (a) Newtonian MD under strain control; (b) Damped system under strain control

(a)

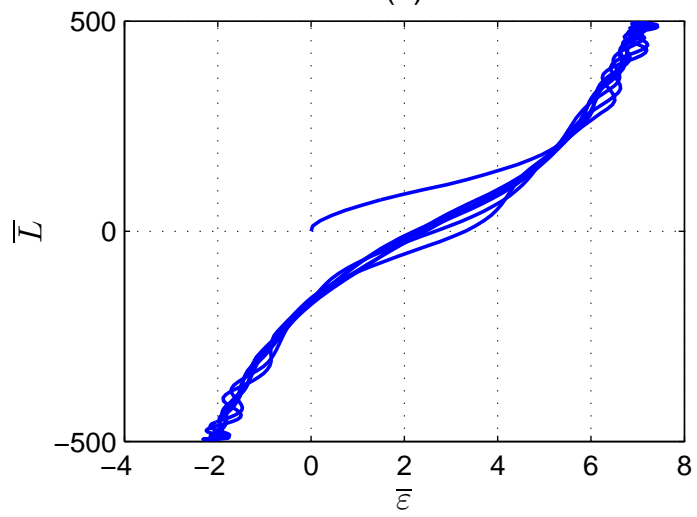

(b)

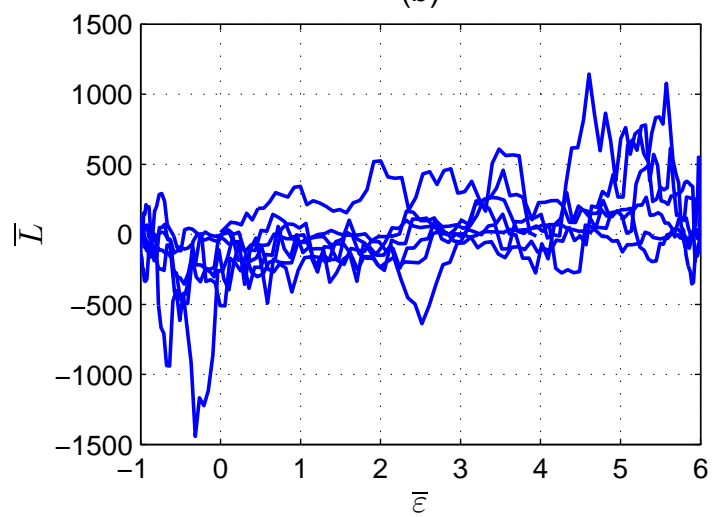

Figure 4: (a) Averaged load-strain curve in Newtonian MD under load control; (b) Averaged load-strain curve in Newtonian MD under strain control 
high when the reference temperature is set high, and with this high kinetic energy in the atomic system, jumping over the double-well energy barrier becomes much easier, even without any phase transformation; On the contrary, when the kinetic energy is forced to be low, significant amount of potential energy is required for the atoms to go over the barrier.

\subsection{DYNAMICS VS. QUASI-STATIC}

In this section, we consider the atomic chain under quasi-static dynamics, i.e. with inertia set to zero. We solve the following system of nonlinear equations,

$$
\begin{aligned}
\frac{\partial \phi}{\partial u_{i}} & =0, \quad \forall i \text { from } 2 \text { to } N-1, \\
\frac{\partial \phi}{\partial u_{N}} & =L .
\end{aligned}
$$

We set $N=100$ in the tests. In the case of load control, $L=A \sin \omega t$. The unknowns $\left(u_{2}, \ldots, u_{N}\right)$ can be solved by using Newton-Raphson method. In the case of strain control, we impose the constrain on the displacement of the last atom at the free end as before, then we solve $\left(u_{2}, \ldots, u_{N-1}\right)$ and $L$. We also run the dynamical systems with small viscosity and with thermostat. The results are exhibited in Figure 7 and 8, respectively. We find that

1. The results from the damped system and thermostatted MD are quite similar to each other.

2. Although the hysteresis in the cases of load control and strain control looks quite different when the loading rate is large, it converges as the loading rate decreases and the two cases give almost the same stress-strain curve.

3. When the loading rate is small, the stress-strain response from the dynamical tests (with damping or a thermostat) is consistent with the quasi-static results in the stable regions (the stable regions correspond to strains or interatomic separations where the potential function is locally convex). In the limit of $\omega \rightarrow 0$, the hysteresis will converge to the quasi-static results and is independent of $\omega$ (see Chapter 3, [1] on a discussion of the static hysteresis based on a continuum theory).

\section{Practical Time Averaging}

This section is mainly adopted from [16] and presented here for completeness.

(a)

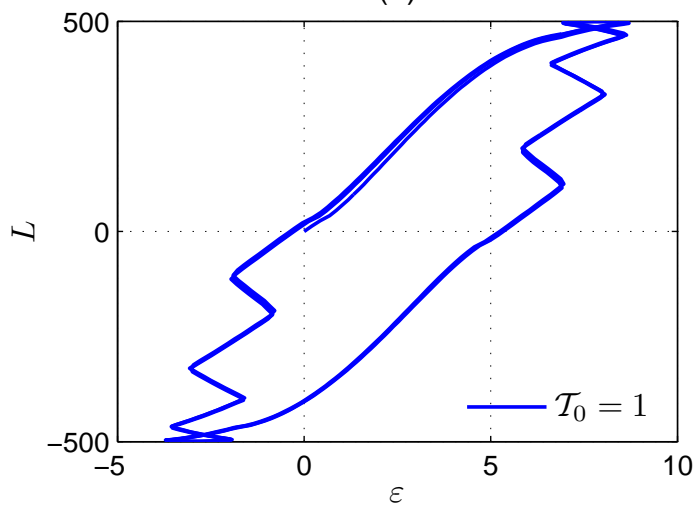

(b)

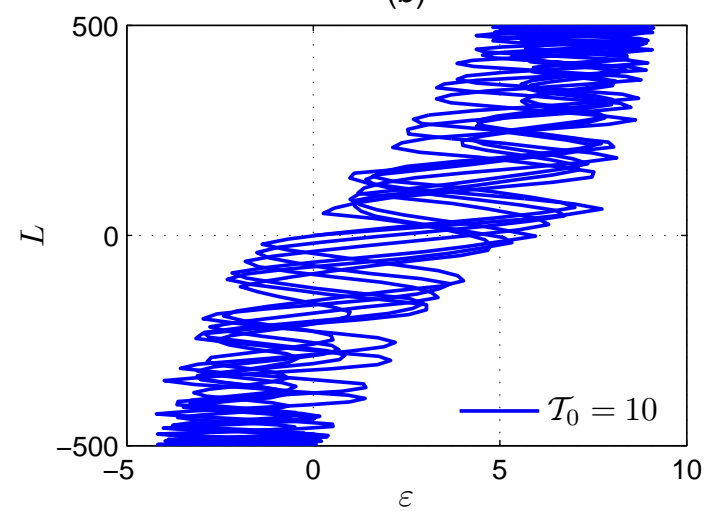

Figure 5: MD with Thermostat under load control 
(a)

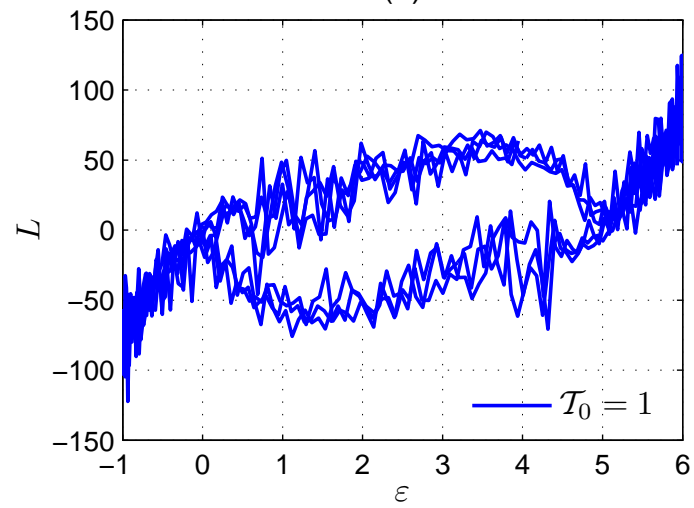

(b)

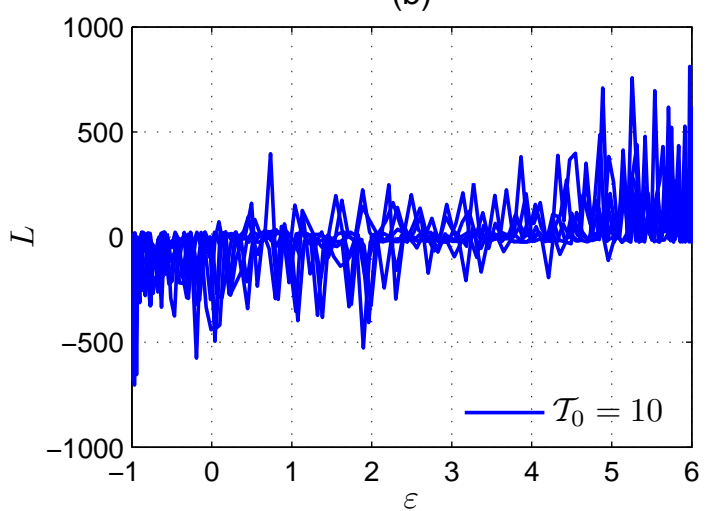

Figure 6: MD with Thermostat under strain control

(a)

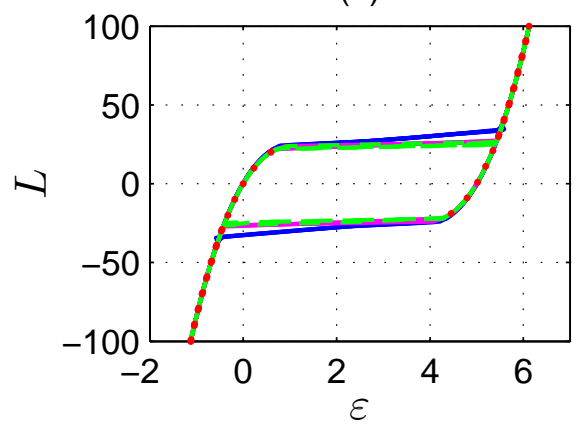

(b)

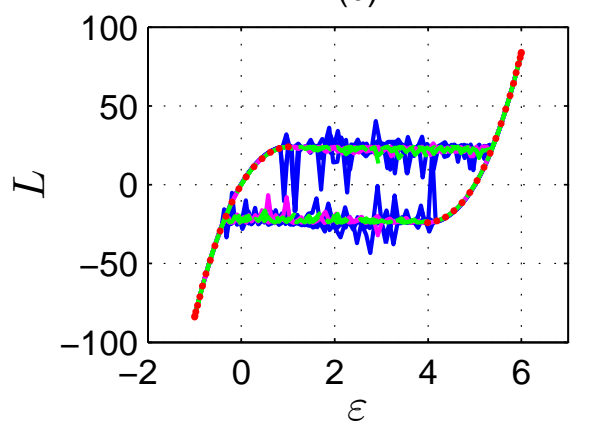

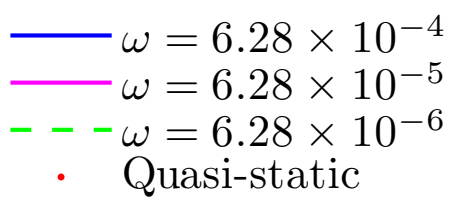

- Quasi-static

Figure 7: Comparison of the hysteresis of damped system and the stress-strain curve from quasi-static loading. (a) Load Control; (b) Strain Control.

(a)

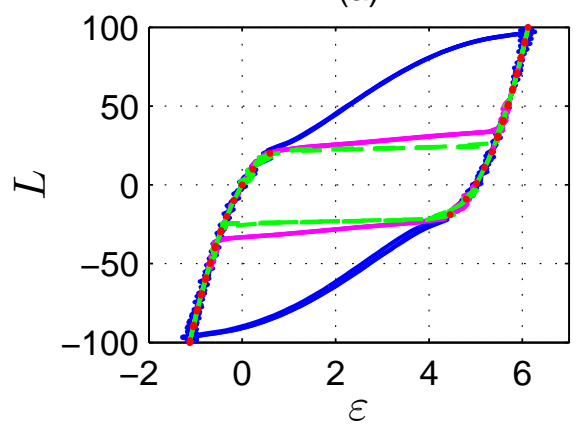

(b)

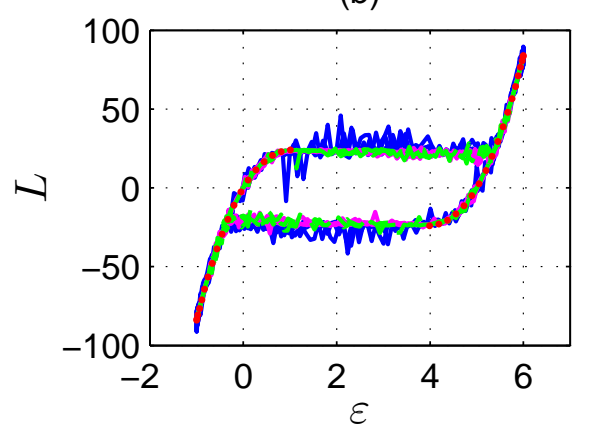

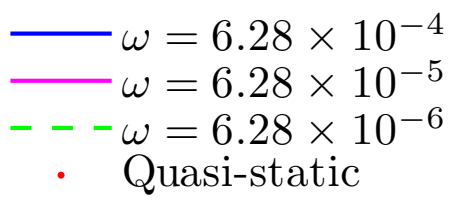

Figure 8: Comparison of the hysteresis of MD with thermostat and the stress-strain curve from quasistatic loading. (a) Load Control; (b) Strain Control. 
The problems of interest here are singularly perturbed systems that display highly oscillatory response and whose limiting behavior often retains fast oscillatory response. Many physical problems can be expected to fall into this category. For instance, in MD simulations where external loading is applied to the system, the loading rate is usually at least $10^{9}$ times slower than the fundamental frequency of atomic vibrations. This large separation of time-scales leads to a singularly perturbed form of the fine-scale dynamics.

The work of [4] building on earlier work of [5] allows dealing with limits of fast flows that are not necessarily equilibria. The theory accounts for the limit behavior of fast flows that may be supported on $\omega$-limit sets of complicated topology. The relevant part of the theorem is stated below, following the terminology presented in [15]:

Suppose the ODEs of the fine-scale dynamics are given as

$$
\begin{aligned}
& \frac{d f}{d t}=H(f, I) \\
& \frac{d I}{d t}=\frac{1}{\mathrm{~T}} L(I),
\end{aligned}
$$

where $f$ is an $N$-dimensional vector of fine degrees of freedom and $H$ is a generally nonlinear function of fine states, referred to as the vector field of the fine dynamical system. $N$ can be large in principle, and the function $H$ rapidly oscillating. $I$ is an $n$ dimensional vector of slow loading variables that do not vary appreciably compared with the oscillation of fine variables. Here, $\mathrm{T}$ is a non-dimensional parameter that represents the ratio of natural frequency of the fine dynamics to the loading frequency, i.e. $\mathrm{T}:=\omega_{f} / \omega$, where $\omega_{f}$ denotes a characteristic frequency of the fast dynamics and $\omega$ is the time scale of the loading (e.g. constant rate of monotonic loading, frequency of cyclic loading). $L$ takes the form $L(I):=\hat{L}(I) \omega_{f}$, where $\hat{L}$ is a bounded smooth function with dimensions of the load vector $I$. For $\omega_{f}$ fixed (which is what we have in mind), by definition, $\mathrm{T} \rightarrow \infty$ as $\omega \rightarrow 0$.

Let $c$ denote the coarse variables of dimension $m$ and $\Lambda$ be a user-specified function of the fine states producing vectors with $m$ components whose time averages over intervals of period $\tau$ can be measured in principle and are of physical interest. Given the fixed time interval $\tau$ characterizing the resolution of coarse measurements in time, a coarse trajectory corresponding to each fine trajectory $f(\cdot)$ is defined as the following running time average:

$$
c(t):=\frac{1}{\tau} \int_{t}^{t+\tau} \Lambda(f(p)) d p
$$

where $\tau<1 / \omega$. Thus, $\tau$ is the period of time averaging, referred to the fast time scale. In this study, we are interested in understanding the evolution of time averages of phase functions of the fast flow on the slow time scale of loading. The developed approach is a natural, practical extension of the method of averaging proposed in [5, 4]. We construct the evolutionary equations of time averaged quantities following the work of [15] and refer to this collection of theoretical and numerical ideas as 'Practical Time Averaging'.

Define a new time scale $s:=t / \mathrm{T}$ and denote $\varepsilon:=1 / \mathrm{T}$. Note that since $\mathrm{T}$ is dimensionless, $s$ has the unit of time and $\varepsilon$ is a dimensionless number. For the functions $f$ and $I$ of the fast time variable $t$, we define the functions $\tilde{f}$ and $\tilde{I}$ of the slow time variable $s$ as follows:

$$
\tilde{f}(s)=f(s \mathrm{~T}), \quad \tilde{I}(s)=I(s \mathrm{~T}) .
$$

Then, on the slow time scale the system reads

$$
\begin{aligned}
& \varepsilon \frac{d \tilde{f}}{d s}=H(\tilde{f}, \tilde{L}) \\
& \frac{d \tilde{I}}{d s}=L(\tilde{I}) .
\end{aligned}
$$


The coarse variable is defined as

$$
\tilde{c}(s)=c(s \mathrm{~T})=\frac{1}{\tau} \int_{s \mathrm{~T}}^{s \mathrm{~T}+\tau} \Lambda(f(p)) d p,
$$

and we assume that $\tau=\kappa / \omega$ with $\kappa$ a non-dimensional constant and $0<\kappa<1$. Introducing the change of variables $r=p / \mathrm{T}$, we have

$$
\tilde{c}(s)=\frac{1}{\lambda} \int_{s}^{s+\lambda} \Lambda(\tilde{f}(r)) d r
$$

where $\lambda=\tau / \mathrm{T}$. Therefore, $\lambda=\kappa /(\omega \mathrm{T})=\kappa / \omega_{f}$ is a constant as $\varepsilon \rightarrow 0$, with physical dimensions of time.

Removing all overhead tildes, we have the slow-fast system, posed on the slow time scale:

$$
\begin{aligned}
& \varepsilon \frac{d f}{d s}=H(f, I) \\
& \frac{d I}{d s}=L(I) \\
& c(s)=\frac{1}{\lambda} \int_{s}^{s+\lambda} \Lambda(f(r)) d r .
\end{aligned}
$$

Let $f^{\varepsilon, I, f_{0}}$ represent solutions of the fast system

$$
\varepsilon \frac{d f^{\varepsilon, I, f_{0}}}{d s}=H\left(f^{\varepsilon, I, f_{0}}, I\right) \quad ; \quad f^{\varepsilon, I, f_{0}}(0)=f_{0}
$$

with $I$ fixed. Rewrite the fast system with the introduction of the fast time scale $t=s / \varepsilon$,

$$
\frac{d f^{(0), I, f_{0}}}{d t}(t)=H\left(f^{(0), I, f_{0}}(t), I\right) ; f^{(0), I, f_{0}}(0)=f_{0}
$$

where

$$
f^{(0), I, f_{0}}(t)=f^{(0), I, f_{0}}\left(\frac{s}{\varepsilon}\right)=f^{\varepsilon, I, f_{0}}(s) .
$$

Assume that for $0 \leq t<\infty, f^{\varepsilon, I, f_{0}}$ lie in a bounded set for each $I$. Hence, on $[0, \mathrm{~T}]$ we will have for any continuous function $F$

$$
F\left(f^{\varepsilon, I, f_{0}}(s)\right) \rightarrow \int_{\mathbb{R}^{N}} F(\gamma) \mu_{s, I, f_{0}}(\gamma) d \gamma \text { weak } \star L^{\infty}[0, \mathrm{~T}],
$$

where $\mu_{s, I, f_{0}}$ is the Young measure generated by the sequence $f^{\varepsilon, I, f_{0}} \in L^{\infty}\left(0, \mathrm{~T} ; \mathbb{R}^{N}\right)$. Moreover, the theory proves that $\mu_{s, I, f_{0}}$ is an invariant measure supported on the $\omega$-limit set of $f^{(0), I, f_{0}}$.

Note that $f^{\varepsilon, I, f_{0}}$ is obtained from running the fast system (42) for a sufficiently long period of time, starting from $f_{0}$ while keeping $I$ fixed; The function $F$ in (44) can be linear or nonlinear. In situation when the $\omega$-limit set of the fast dynamics is not an equilibrium point, the Tikhonov approach may not in general be expected to approximate the time evolution of the first-moment of nonlinear functions of state. Furthermore, the theory proves that coarse evolution equations can be written down for state variables that are 'slow', i.e. orthogonal to the fast flow in a precise sense, but does not provide a prescription for generating such 'orthogonal observables'.

We follow the work of [15], which provides a straightforward way of generating a large class of slow variables via time averaging that are shown to be 'orthogonal observables' (for a corresponding infinite dimensional dynamics) for which a slow dynamics can be written down. This approach and the numerical approximation ideas developed here are referred as 'Practical Time Averaging'. 
Recall the coarse variable defined on the slow time scale (40) and write down its evolution:

$$
c(s)=\frac{1}{\lambda} \int_{s}^{s+\lambda} \Lambda(f(r)) d r \Longrightarrow \frac{d c}{d s}(s)=\frac{1}{\lambda}[\Lambda(f(s+\lambda))-\Lambda(f(s))] .
$$

Let us define the sequence (on $\varepsilon$ ) of smooth functions $c^{\varepsilon, f_{0}}$ such that

$$
c^{\varepsilon, f_{0}}(s)=\frac{1}{\lambda} \int_{s}^{s+\lambda} \Lambda\left(f^{\varepsilon, I(r), f_{0}}(r)\right) d r
$$

where we follow the notation in (41) but now $I$ is a function of the slow time. Then the evolution equation is

$$
\frac{d c^{\varepsilon, f_{0}}}{d s}(s)=\frac{1}{\lambda}\left[\Lambda\left(f^{\varepsilon, I(s+\lambda), f_{0}}(s+\lambda)\right)-\Lambda\left(f^{\varepsilon, I(s), f_{0}}(s)\right)\right] .
$$

Let $\bar{c}^{f_{0}}$ denote the weak limit of the sequence $c^{\varepsilon, f_{0}}$, and using (44), we have

$$
\frac{d \bar{c}^{f_{0}}}{d s}=\frac{1}{\lambda}\left(\int_{\mathbb{R}^{N}} \Lambda(\gamma) \mu_{s+\lambda, I(s+\lambda), f_{0}}(\gamma) d \gamma-\int_{\mathbb{R}^{N}} \Lambda(\gamma) \mu_{s, I(s), f_{0}}(\gamma) d \gamma\right)
$$

in the weak (variational) sense. (48) can be considered as the instantaneous evolution equation of the limit slow variable $\bar{c}^{f_{0}}$ of the sequence $c^{\varepsilon, f_{0}}$. It should be noted that if any arbitrary instantaneous function, say $A(f)$, is taken as the coarse variable, we have

$$
\frac{d A(f)}{d s}(s)=\frac{1}{\varepsilon} \nabla_{f} A \cdot H .
$$

Since $1 / \varepsilon$ is built in the evolution equation, the Young measure limit is usually not applicable unless the function $A(f)$ satisfies $\nabla_{f} A \cdot H=0$. However, our definition of coarse variables allows constructing coarse evolution equation for more general kinds of averaged phase functions. For example, to approximate the change of temperature of a MD system, where temperature can be interpreted as a function of particle velocities but normally does not satisfy $\nabla_{f} A \cdot H=0$, we can define the time average of temperature and follow the procedure from (45) to (48) to write down the coarse evolution equation.

The Young measures in (48) are approximated as averages of $M$ Dirac masses at $M$ values of $f$, i.e.

$$
\mu_{s, I(s), f_{0}} \approx \frac{1}{M} \sum_{i=1}^{M} \delta\left(f^{(0), I(s), f_{0}}\left(t_{i}\right)\right),
$$

where $t_{i}$ is a discrete time instant in the fast run from i.c. $f_{0}$ and $I(s)$ held fixed. $M$ is normally chosen to be large enough for the averages to converge. For practical numerics we have found that a 'direct' implementation of (48) does not work. In the following we discuss what a 'direct' implementation might constitute and the actual procedure that we adopt.

The procedures of the 'direct' implementation suggested in [6] include:

1. Evolve the fast system (42) with slow variables fixed at time step $s$ and $s+\lambda$, respectively to estimate the weak limit of the continuous function $\Lambda$, i.e.

$$
\begin{aligned}
& \int_{\mathbb{R}^{N}} \Lambda(\gamma) \mu_{s, I(s), f_{0}}(\gamma) d \gamma \approx \frac{1}{M} \sum_{i=1}^{M} \Lambda\left(f^{(0), I(s), f_{0}}\left(t_{i}\right)\right) \\
& \int_{\mathbb{R}^{N}} \Lambda(\gamma) \mu_{s+\lambda, I(s+\lambda), f_{0}}(\gamma) d \gamma \approx \frac{1}{M} \sum_{i=1}^{M} \Lambda\left(f^{(0), I(s+\lambda), f_{0}}\left(t_{i}\right)\right),
\end{aligned}
$$

where the above equations are derived from (50). 
2. Extrapolate to obtain the coarse variable at the next coarse step $s+T$ using the Forward Euler method,

$$
\bar{c}^{f_{0}}(s+T) \approx \bar{c}^{f_{0}}(s)+\frac{T}{\lambda}\left[\int_{\mathbb{R}^{N}} \Lambda(\gamma) \mu_{s+\lambda, I(s+\lambda), f_{0}}(\gamma) d \gamma-\int_{\mathbb{R}^{N}} \Lambda(\gamma) \mu_{s, I(s), f_{0}}(\gamma) d \gamma\right],
$$

where $T$ is the coarse time step. Usually $T$ is set to be much larger than $\lambda . I(s+T)$ can be obtained from evolving $(40)_{2}$ solely.

3. Reconstruct to find fine data that matches $\bar{c}^{f_{0}}(s+T)$ and $I(s+T)$.

4. Repeat.

The primary shortcoming of all of this theory as acknowledged in $[5,4]$ and carries over for the model with time averages in [15] is that the theory is silent about fine initial conditions required to generate the Young measures at the discrete slow time-levels. This is a serious practical issue and renders $f_{0}$ as an essentially free parameter whose choice, nevertheless, affects results and has to be made on an ad-hoc basis based on numerical experimentation. We mention here that the unsubstantiated assumption of ergodicity is often made to sweep this important issue under the rug.

Given the above uncertainty and the need to use it twice to follow the evolution prescribed by (52), an alternative procedure is the following. Based on (43), the sequence of functions $c^{\varepsilon, f_{0}}(s)$ can be written as

$$
c^{\varepsilon, f_{0}}(s)=c^{(0), f_{0}}\left(\frac{s}{\varepsilon}\right)=c^{(0), f_{0}}(t) .
$$

Using the substitutions $p=r / \varepsilon, \tau=\lambda / \varepsilon$ and (43), the right-hand-side term in (46) can be interpreted on the fast time scale as

$$
\frac{1}{\lambda} \int_{s}^{s+\lambda} \Lambda\left(f^{\varepsilon, I(r), f_{0}}(r)\right) d r \approx \frac{1}{\tau} \int_{t}^{t+\tau} \Lambda\left(f^{(0), I(t), f_{0}}(p)\right) d p
$$

where we make the realistic assumption that the loads $I$ do not vary appreciably in the (fasttime) interval $[t, t+\tau]$. From (53) and (54), we have

$$
c^{(0), f_{0}}(t)=\frac{1}{\tau} \int_{t}^{t+\tau} \Lambda\left(f^{(0), I(t), f_{0}}(p)\right) d p
$$

We are interested in $c^{\varepsilon, f_{0}}$ as $\varepsilon \rightarrow 0$, which by definition, is the time average of functions of the fast variables as $\tau \rightarrow \infty$ when the slow variable $I$ is fixed. We simply follow (55) to calculate this average at each coarse time step $T$. Theoretically, we need to run the fast system for an infinite period of time. However, for the numerical tests conducted here we have seen that the average converges quickly, thus taking a reasonable period of time is sufficient.

\section{2D Ni-Mn System}

The setting of this problem is from [9], which is a 2D lattice system made of Ni-Mn. The initial configuration of the 2D Ni-Mn lattice is shown in Figure 9.

The 2D lattice is infinite in the horizontal direction and has height $H$ in the vertical direction. Since it is infinite, we apply periodic boundary conditions (PBC) on a simulation box, where the total number of atoms (including $\mathrm{Ni}$ and $\mathrm{Mn}$ ) is 1390 . The distance between the twin boundary and the bottom is $h$. In the tests, we set $h=2 / 3 H$. The material above the twin boundary is associated with martensite variant- 1 while the material below it is associated with variant-2. The force $F$ applied on the upper and lower sides of the lattice in the horizontal 


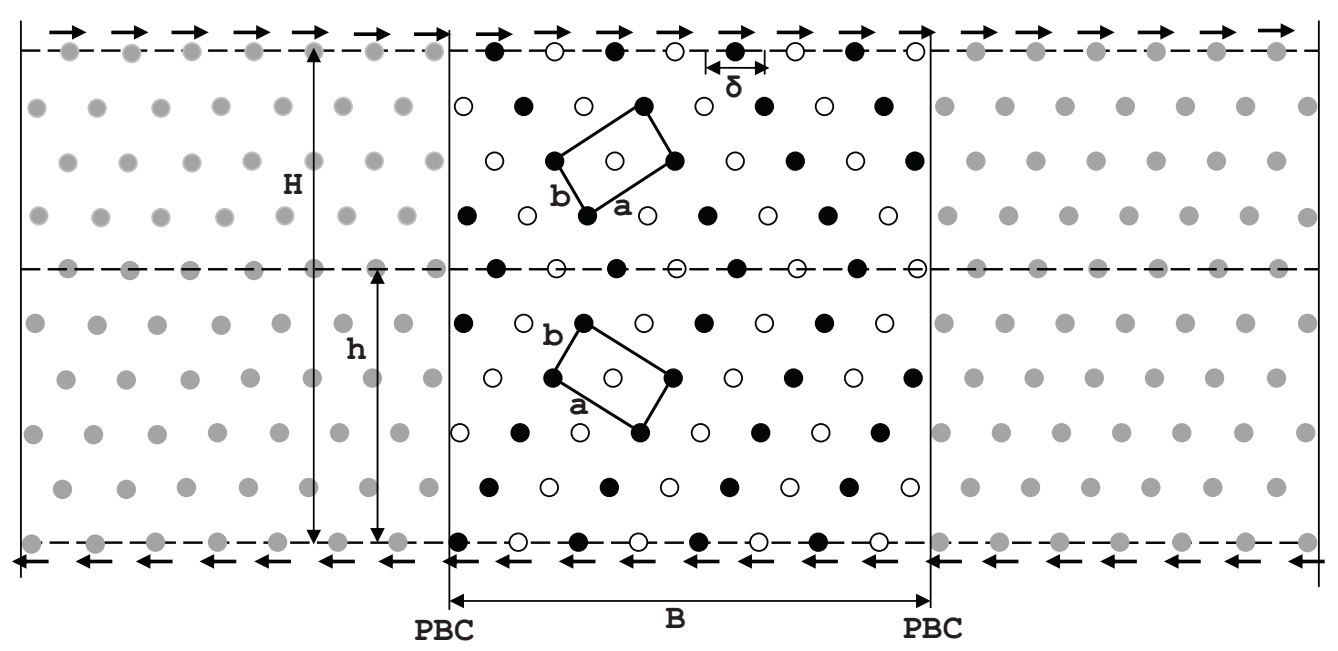

Figure 9: Two-dimensional Ni-Mn lattice of infinite width in the horizontal direction.

direction. Define the domain of the simulation box as $\Omega$. The upper edge of the box is denoted as $\Omega_{\text {upper }}$ and the lower edge is denoted as $\Omega_{\text {lower }}$. We apply strain-control on the upper and lower boundaries of the system, thus the equations of motion are

$$
\begin{aligned}
& \dot{p}_{\alpha i}^{\mathrm{Ni}}=v_{\alpha i}^{\mathrm{Ni}}, \forall \alpha \in \Omega \backslash\left(\Omega_{\text {upper }} \cup \Omega_{\text {lower }}\right), \\
& \dot{p}_{\alpha i}^{\mathrm{Mn}}=v_{\alpha i}^{\mathrm{Mn}}, \forall \alpha \in \Omega \backslash\left(\Omega_{\text {upper }} \cup \Omega_{\text {lower }}\right), \\
& \dot{v}_{\alpha i}^{\mathrm{Ni}}=\frac{1}{m_{\mathrm{Ni}}}\left(-\frac{\partial \Phi}{\partial p_{\alpha i}^{\mathrm{Ni}}}\right), \forall \alpha \in \Omega \backslash\left(\Omega_{\text {upper }} \cup \Omega_{\text {lower }}\right), \\
& \dot{v}_{\alpha i}^{\mathrm{Mn}}=\frac{1}{m_{\mathrm{Mn}}}\left(-\frac{\partial \Phi}{\partial p_{\alpha i}^{\mathrm{Mn}}}\right), \forall \alpha \in \Omega \backslash\left(\Omega_{\text {upper }} \cup \Omega_{\text {lower }}\right), \\
& \dot{p}_{\alpha i}^{\mathrm{Ni}}=\omega, \forall \alpha \in \Omega_{\text {upper }}, \\
& \dot{p}_{\alpha i}^{\mathrm{Mn}}=\omega, \forall \alpha \in \Omega_{\text {upper }}, \\
& \dot{p}_{\alpha i}^{\mathrm{Ni}}=-\omega, \forall \alpha \in \Omega_{\text {lower }}, \\
& \dot{p}_{\alpha i}^{\mathrm{Mn}}=-\omega, \forall \alpha \in \Omega_{\text {lower }} .
\end{aligned}
$$

where $\Phi$ is the total potential energy of the system and $\omega$ is the loading rate. As before, $\alpha$ denotes the atom number and $i$ indicates the directions $(i=1$ for horizontal axis and $i=2$ for vertical axis). We assume Lennard-Jones potentials among the atoms, i.e.,

$$
\Phi^{\alpha \beta}(d)=4 \varepsilon^{\alpha \beta}\left[\left(\frac{\sigma^{\alpha \beta}}{d}\right)^{12}-\left(\frac{\sigma^{\alpha \beta}}{d}\right)^{6}\right],
$$

where $\alpha \beta=\mathrm{NiNi}, \mathrm{MnMn}$ and NiMn. 
Substituting the explicit form of potential energy into (56), we have

$$
\begin{aligned}
& \dot{p}_{\alpha i}^{\mathrm{Ni}}=v_{\alpha i}^{\mathrm{Ni}}, \forall \alpha \in \Omega \backslash\left(\Omega_{\text {upper }} \cup \Omega_{\text {lower }}\right), \\
& \dot{p}_{\alpha i}^{\mathrm{Mn}}=v_{\alpha i}^{\mathrm{Mn}}, \forall \alpha \in \Omega \backslash\left(\Omega_{\text {upper }} \cup \Omega_{\text {lower }}\right), \\
& \dot{v}_{\alpha i}^{\mathrm{Ni}}=\frac{1}{m_{\mathrm{Ni}}}\left\{\begin{array}{l}
-\sum_{\beta^{\mathrm{Ni}} \in\left(\alpha^{\mathrm{Ni}}, r\right)} 24 \varepsilon^{\mathrm{NiNi}}\left[\frac{\left(\sigma^{\mathrm{NiNi}}\right)^{6}}{\left(d_{\alpha \beta}^{\mathrm{NiNi}}\right)^{7}}-2 \frac{\left(\sigma^{\mathrm{NiNi}}\right)^{12}}{\left(d_{\alpha \beta}^{\mathrm{NiNi}}\right)^{13}}\right] \frac{p_{\alpha i}^{\mathrm{Ni}}-p_{\beta i}^{\mathrm{Ni}}}{d_{\alpha \beta}^{\mathrm{NiNi}}} \\
-\sum_{\beta^{\mathrm{Mn}} \in\left(\alpha^{\mathrm{Ni}}, r\right)} 24 \varepsilon^{\mathrm{NiMn}}\left[\frac{\left(\sigma^{\mathrm{NiMn}}\right)^{6}}{\left(d_{\alpha \beta}^{\mathrm{NiMn}}\right)^{7}}-2 \frac{\left(\sigma^{\mathrm{NiMn}}\right)^{12}}{\left(d_{\alpha \beta}^{\mathrm{NiMn}}\right)^{13}}\right] \frac{p_{\alpha i}^{\mathrm{Ni}}-p_{\beta i}^{\mathrm{Mn}}}{d_{\alpha \beta}^{\mathrm{NiMn}}}
\end{array}\right\},
\end{aligned}
$$

$\forall \alpha \in \Omega \backslash\left(\Omega_{\text {upper }} \cup \Omega_{\text {lower }}\right)$,

$\dot{v}_{\alpha i}^{\mathrm{Mn}}=\frac{1}{m_{\mathrm{Mn}}}\left\{\begin{array}{l}-\sum_{\beta^{\mathrm{Mn}} \in\left(\alpha^{\mathrm{Mn}}, r\right)} 24 \varepsilon^{\mathrm{MnMn}}\left[\frac{\left(\sigma^{\mathrm{MnMn}}\right)^{6}}{\left.\left(d_{\alpha \beta}^{\mathrm{MnMn}}\right)^{7}-2 \frac{\left(\sigma^{\mathrm{MnMn}}\right)^{12}}{\left(d_{\alpha \beta}^{\mathrm{MnMn}}\right)^{13}}\right] \frac{p_{\alpha i}^{\mathrm{Mn}}-p_{\beta i}^{\mathrm{Mn}}}{d_{\alpha \beta}^{\mathrm{MnMn}}}}\right. \\ -\sum_{\beta^{\mathrm{Ni}} \in\left(\alpha^{\mathrm{Mn}}, r\right)} 24 \varepsilon^{\mathrm{NiMn}}\left[\frac{\left(\sigma^{\mathrm{NiMn}}\right)^{6}}{\left(d_{\alpha \beta}^{\mathrm{NiMn}}\right)^{7}}-2 \frac{\left(\sigma^{\mathrm{NiMn}}\right)^{12}}{\left(d_{\alpha \beta}^{\mathrm{NiMn}}\right)^{13}}\right] \frac{p_{\alpha i}^{\mathrm{Mn}}-p_{\beta i}^{\mathrm{Ni}}}{d_{\alpha \beta}^{\mathrm{NiMn}}}\end{array}\right\}$,

$\forall \alpha \in \Omega \backslash\left(\Omega_{\text {upper }} \cup \Omega_{\text {lower }}\right)$,

$\dot{p}_{\alpha i}^{\mathrm{Ni}}=\omega, \forall \alpha \in \Omega_{\text {upper }}$,

$\dot{p}_{\alpha i}^{\mathrm{Mn}}=\omega, \forall \alpha \in \Omega_{\mathrm{upper}}$,

$\dot{p}_{\alpha i}^{\mathrm{Ni}}=-\omega, \forall \alpha \in \Omega_{\text {lower }}$,

$\dot{p}_{\alpha i}^{\mathrm{Mn}}=-\omega, \forall \alpha \in \Omega_{\text {lower }}$.

We further define the following non-dimensionalized terms,

$$
\begin{aligned}
& \bar{m}_{\mathrm{Ni}}=\frac{m_{\mathrm{Ni}}}{\mu_{0}}=58.59 ; \quad \bar{m}_{\mathrm{Mn}}=\frac{m_{\mathrm{Mn}}}{\mu_{0}}=54.93 ; \bar{\omega}=\omega T_{0} \\
& \bar{\varepsilon}^{\mathrm{NiNi}}=\frac{\varepsilon^{\mathrm{NiNi}}}{\varepsilon_{0}}=1.0 ; \quad \bar{\varepsilon}^{\mathrm{NiMn}}=\frac{\varepsilon^{\mathrm{NiMn}}}{\varepsilon_{0}}=0.9905 ; \quad \bar{\varepsilon}^{\mathrm{MnMn}}=\frac{\varepsilon^{\mathrm{MnMn}}}{\varepsilon_{0}}=0.9810 ; \\
& \bar{\sigma}^{\mathrm{NiNi}}=\frac{\sigma^{\mathrm{NiNi}}}{\sigma_{0}}=1.0 ; \quad \bar{\sigma}^{\mathrm{NiMn}}=\frac{\sigma^{\mathrm{NiMn}}}{\sigma_{0}}=1.0205 ; \quad \bar{\sigma}^{\mathrm{MnMn}}=\frac{\sigma^{\mathrm{MnMn}}}{\sigma_{0}}=0.8736 .
\end{aligned}
$$

The parameters $\varepsilon_{0}, \sigma_{0}, \mu_{0}$ and $T_{0}$ are chosen following [9], i.e.,

$$
\varepsilon_{0}=\varepsilon^{\mathrm{NiNi}} ; \sigma_{0}=\sigma^{\mathrm{NiNi}} ; \mu_{0}=1.66011 \times 10^{-27} \mathrm{~kg} ; T_{0}=\sqrt{\mu_{0} \sigma_{0}^{2} / \varepsilon_{0}} .
$$


The non-dimensionalized equations of motion become

$$
\begin{aligned}
& \left(\bar{p}_{\alpha i}^{\mathrm{Ni}}\right)^{\prime}=\bar{v}_{\alpha i}^{\mathrm{Ni}}, \forall \alpha \in \Omega \backslash\left(\Omega_{\text {upper }} \cup \Omega_{\text {lower }}\right), \\
& \left(\bar{p}_{\alpha i}^{\mathrm{Mn}}\right)^{\prime}=\bar{v}_{\alpha i}^{\mathrm{Mn}}, \forall \alpha \in \Omega \backslash\left(\Omega_{\text {upper }} \cup \Omega_{\text {lower }}\right) \text {, } \\
& \left(\bar{v}_{\alpha i}^{\mathrm{Ni}}\right)^{\prime}=\frac{1}{\bar{m}_{\mathrm{Ni}}}\left\{\begin{array}{l}
-\sum_{\beta^{\mathrm{Ni}} \in\left(\alpha^{\mathrm{Ni}}, r\right)} 24 \bar{\varepsilon}^{\mathrm{NiNi}}\left[\frac{\left(\bar{\sigma}^{\mathrm{NiNi}}\right)^{6}}{\left(\bar{d}_{\alpha \beta}^{\mathrm{NiNi}}\right)^{7}}-2 \frac{\left(\bar{\sigma}^{\mathrm{NiNi}}\right)^{12}}{\left(\bar{d}_{\alpha \beta}^{\mathrm{NiNi}}\right)^{13}}\right] \frac{\bar{p}_{\alpha i}^{\mathrm{Ni}}-\bar{p}_{\beta i}^{\mathrm{Ni}}}{\bar{d}_{\alpha \beta}^{\mathrm{NiNi}}} \\
-\sum_{\beta^{\mathrm{Mn}} \in\left(\alpha^{\mathrm{Ni}}, r\right)} 24 \bar{\varepsilon}^{\mathrm{NiMn}}\left[\frac{\left(\bar{\sigma}^{\mathrm{NiMn}}\right)^{6}}{\left(\bar{d}_{\alpha \beta}^{\mathrm{NiMn}}\right)^{7}}-2 \frac{\left(\bar{\sigma}^{\mathrm{NiMn}}\right)^{12}}{\left(\bar{d}_{\alpha \beta}^{\mathrm{NiMn}}\right)^{13}}\right] \frac{\bar{p}_{\alpha i}^{\mathrm{Ni}}-\bar{p}_{\beta i}^{\mathrm{Mn}}}{\bar{d}_{\alpha \beta}^{\mathrm{NiMn}}}
\end{array}\right\}, \\
& \forall \alpha \in \Omega \backslash\left(\Omega_{\text {upper }} \cup \Omega_{\text {lower }}\right) \text {, } \\
& \left(\bar{v}_{\alpha i}^{\mathrm{Mn}}\right)^{\prime}=\frac{1}{\bar{m}_{\mathrm{Mn}}}\left\{\begin{array}{l}
-\sum_{\beta^{\mathrm{Mn}} \in\left(\alpha^{\mathrm{Mn}}, r\right)} 24 \bar{\varepsilon}^{\mathrm{MnMn}}\left[\frac{\left(\bar{\sigma}^{\mathrm{MnMn}}\right)^{6}}{\left(\bar{d}_{\alpha \beta}^{\mathrm{MnMn}}\right)^{7}}-2 \frac{\left(\bar{\sigma}^{\mathrm{MnMn}}\right)^{12}}{\left(\bar{d}_{\alpha \beta}^{\mathrm{MnMn}}\right)^{13}}\right] \frac{\bar{p}_{\alpha i}^{\mathrm{Mn}}-\bar{p}_{\beta i}^{\mathrm{Mn}}}{\bar{d}_{\alpha \beta}^{\mathrm{MnMn}}} \\
-\sum_{\beta^{\mathrm{Ni}} \in\left(\alpha^{\mathrm{Mn}}, r\right)} 24 \bar{\varepsilon}^{\mathrm{NiMn}}\left[\frac{\left(\bar{\sigma}^{\mathrm{NiMn}}\right)^{6}}{\left(\bar{d}_{\alpha \beta}^{\mathrm{NiMn}}\right)^{7}}-2 \frac{\left(\bar{\sigma}^{\mathrm{NiMn}}\right)^{12}}{\left(\bar{d}_{\alpha \beta}^{\mathrm{NiMn}}\right)^{13}}\right] \frac{\bar{p}_{\alpha i}^{\mathrm{Mn}}-\bar{p}_{\beta i}^{\mathrm{Ni}}}{\bar{d}_{\alpha \beta}^{\mathrm{NiMn}}}
\end{array}\right\}, \\
& \forall \alpha \in \Omega \backslash\left(\Omega_{\text {upper }} \cup \Omega_{\text {lower }}\right) \text {, } \\
& \left(\bar{p}_{\alpha i}^{\mathrm{Ni}}\right)^{\prime}=\bar{\omega}, \forall \alpha \in \Omega_{\text {upper }}, \\
& \left(\bar{p}_{\alpha i}^{\mathrm{Mn}}\right)^{\prime}=\bar{\omega}, \forall \alpha \in \Omega_{\text {upper }}, \\
& \left(\bar{p}_{\alpha i}^{\mathrm{Ni}}\right)^{\prime}=-\bar{\omega}, \forall \alpha \in \Omega_{\text {lower }}, \\
& \left(\bar{p}_{\alpha i}^{\mathrm{Mn}}\right)^{\prime}=-\bar{\omega}, \forall \alpha \in \Omega_{\text {lower }} .
\end{aligned}
$$

In the following discussion, the overbars are removed in the above equations for simplicity. The numerical tests are carried out using LAMMPS [13]. The initial configurations are set to be in force equilibrium at $0 \mathrm{~K}$, thus the particle velocities are zero in the beginning. We use the values from [9] to create the geometry of the lattice, that are

$$
a=1.54110 \sigma_{0}, \quad b=1.14101 \sigma_{0} .
$$

The force equilibrium can be obtained by minimization. Following the initial set-up, we run NVE ensemble (i.e. with specifying macroscopic variables being the number of particles $N$, the volume $V$ and the total energy $E$ ) to generate the fine trajectories of the system. The temperature is small during the evolution but we do NOT employ a thermostat.

Figure 10(a) shows the stress-strain curve from fine dynamics. The displacement $u$ and the loading $L$ are defined as

$$
u=\frac{u_{\text {upper }}-u_{\text {lower }}}{2},
$$

and

$$
L=\frac{L_{\text {upper }}-L_{\text {lower }}}{2},
$$

respectively, where $u_{\text {upper }}$ and $u_{\text {lower }}$ indicate the space-averaged displacements of the upper and lower atoms. The loading $L$ is the average of the magnitudes of the load applied on the top and bottom surfaces. The shear strain $\gamma$ and shear stress $\tau$ are defined as follows

$$
\gamma=\frac{2 u}{H}, \quad \tau=\frac{L}{B \times b},
$$


where $H$ and $B$ are the height and width of the simulation box as indicated in Figure $9 . \quad b$ is the nearest distance between two $\mathrm{Ni}$, which is considered as the width in the third direction.

We have tested three loading rates, i.e. $\omega=10^{-3}, 10^{-4}$ and $10^{-5}$. The characteristic frequency of atomic system is about 1 (note that we are dealing with (61), thus the approximated quantity is dimensionless), so the loading rate is small compared to atomic oscillations. The hysteresis is obvious in all cases. The twin boundary nucleates at different places and the variant-2 is gradually transformed to variant-1, which is associated with lower free energies. At this stage, the deformation under shearing is accommodated by the solid phase transformation, which corresponds to the softening part in the stress-strain curve. Once the variant-2 has been fully transformed to variant-1, the lattice deforms like a homogeneous body under shearing, which gives linear-elastic stress-strain behavior. During the unloading process, the lattice first recovers from shear deformation, followed by phase transformation from variant- 1 to variant- 2 . Once the lattice consists of all variant-2 of martensite, it will again deform homogenously. In the implementation of PTA, the procedures are as follows based on the theory given in Section 3 :

1. To begin with, we need the fine-scale problem defined as a system of autonomous ODE, i.e.(61). The initial conditions (such as atom positions and velocities) also need to be specified. In this case, we choose crystals at $0 \mathrm{~K}$.

2. Starting from the given initial conditions, we run the fast system (i.e. MD) for a sufficiently long period of time to generate the Young measures while holding the slow variables (i.e. the external loading) fixed. The running time is chosen until the Young measure converges.

3. The coarse variables of interest (in this case, we choose shear strain and stress defined in (65)) are computed using (55) at $s=0$.

4. Next, we need to take a large coarse time step $T$ to generate the Young measures at $s=T$, which depends heavily on the fast initial conditions $f_{0}$. Here we assume the initial conditions to be the last state from the fine trajectories in the Young measure approximant run in the previous coarse time step. Since the shear force is applied horizontally, it is reasonable to assume that the movement of the particles in the vertical direction is negligible. Therefore, the vertical atom velocities are reset to be zero in the initial conditions for each Young measure approximant run.

5. The steps 2-4 are repeated until we obtain the coarse dynamics for a desirable period of time.

Figure 10(b) exhibits the stress-strain curves from PTA with different coarse time steps. It can be seen that setting $T=0.5$ gives considerably different result from the other two while the curves from $T=0.2$ and $T=0.1$ are quite similar. In Figure 11, we compare the results from fine dynamics and from PTA. The loading rate is $10^{-4}$ in fine dynamics. The response from PTA in the case of $T=0.2$ is presented in Figure 11(a). It seems that PTA is able to predict the softening and linear-elastic parts of the hysteresis very well. Figure 11(b) demonstrates coarse dynamics from PTA with other options for defining the initial conditions. However, these approximations are not as good as before. Figure 12 presents snapshots for one full cycle of sinusoidal loading from direct numerical simulation of MD and from configurations recovered from Young measure approximant runs from Figure 11(a). We can easily observe the process of phase transformation between variant-1 and variant-2. Although the atomic configurations diverge locally, PTA succeeds in approximating the overall behavior of the 2D lattice under shear deformation. If taking a more detailed view of the transformation of individual atoms on a row at different times, we observe the motion of steps along a twin boundary in the horizontal direction, both from fine and PTA. The observation of step motions is also discussed in $[9,18,12]$. 
A rough estimation of the total steps in the time integration of the fine ODE is the following

$$
\begin{aligned}
n_{f} & =\frac{T_{\mathrm{tot}}}{\omega \triangle t}, \\
n_{s} & =\frac{T_{\mathrm{tot}}}{T} \times M .
\end{aligned}
$$

In the above equations, $n_{f}$ and $n_{s}$ denote the total time steps required to run the full fine ODE over the total simulation time and the time steps required to generate Young measure approximants (i.e. (50)) in all coarse steps when implementing PTA. $T_{\text {tot }}$ is the total simulation time defined on the slow time scale. $\triangle t$ is the fast time step and $M$ is the number of integration steps needed in the approximation of Young measures in each coarse step. The parameters we used in the numerical simulations are $\Delta t=0.01$ and $M=1000$. Thus in the case of Figure 11(a), the total time savings in PTA is about 200 times of fine.

\section{3D Atomic Model}

Figure 13 shows the initial configuration of the atomic assembly. We are simulating a cubic lattice with lattice spacing $a_{s}$ where each cube is face-centered. For each atom $\alpha$, its position and momentum vectors have components in $x, y$ and $z$ directions, respectively, i.e.,

$$
\underline{p}_{\alpha}=\left(p_{\alpha 1}, p_{\alpha 2}, p_{\alpha 3}\right)^{T}, \underline{q}_{\alpha}=\left(q_{\alpha 1}, q_{\alpha 2}, q_{\alpha 3}\right)^{T} .
$$

The system is under uniaxial tension along the longitudinal direction. The atoms are at rest initially. The interatomic interactions between any pair of atoms are modeled by Lennard-Jones (LJ) potentials. Similar to (61), the non-dimensionalized Hamiltonian equation is given by

$$
\begin{aligned}
& \left(\bar{p}_{\alpha i}\right)^{\prime}=\bar{v}_{\alpha i}, \forall \alpha \in \Omega \backslash\left(\Omega_{\text {left }} \cup \Omega_{\text {right }}\right), \\
& \left(\bar{v}_{\alpha i}\right)^{\prime}=-\frac{1}{\bar{m}} \sum_{\beta \in(\alpha, r)} 24 \bar{\varepsilon}\left[\frac{(\bar{\sigma})^{6}}{\left(\bar{d}_{\alpha \beta}\right)^{7}}-2 \frac{(\bar{\sigma})^{12}}{\left(\bar{d}_{\alpha \beta}\right)^{13}}\right] \frac{\bar{p}_{\alpha i}-\bar{p}_{\beta i}}{\bar{d}_{\alpha \beta}} \forall \alpha \in \Omega \backslash\left(\Omega_{\text {left }} \cup \Omega_{\text {right }}\right), \\
& \left(\bar{p}_{\alpha i}\right)^{\prime}=-\bar{\omega}, \forall \alpha \in \Omega_{\text {left }}, \\
& \left(\bar{p}_{\alpha i}\right)^{\prime}=\bar{\omega}, \forall \alpha \in \Omega_{\text {right }} .
\end{aligned}
$$

(a)

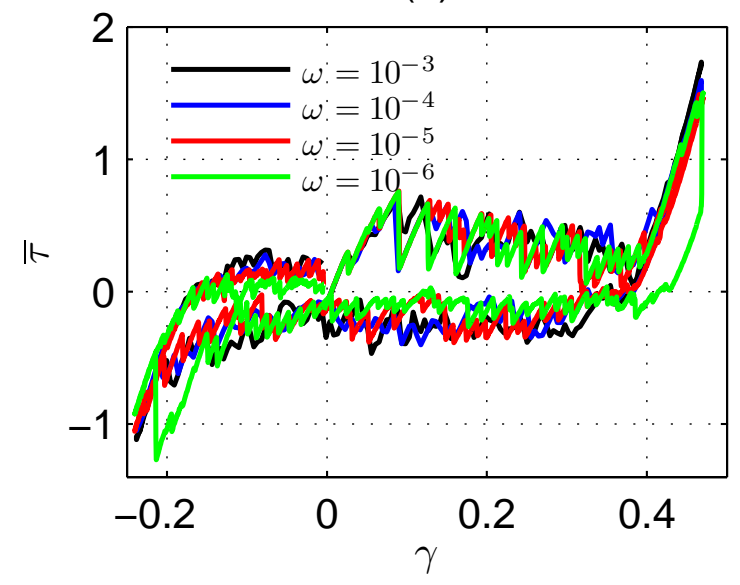

(b)

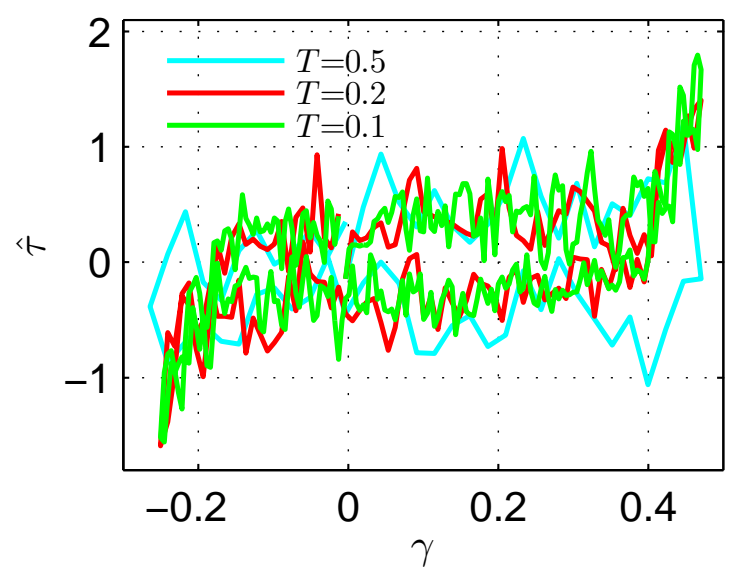

Figure 10: (a) Stress-strain curve from fine dynamics with different loading frequencies; (b) Stress-strain curve from PTA with different choices of coarse time steps. 
(a)

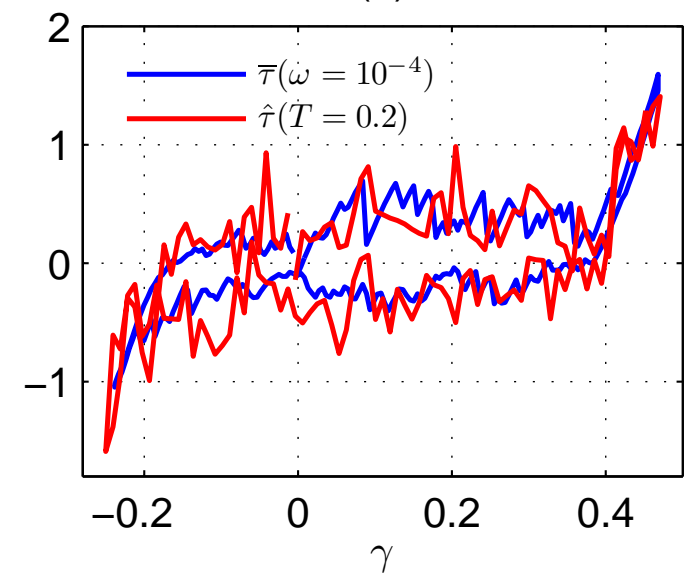

(b)

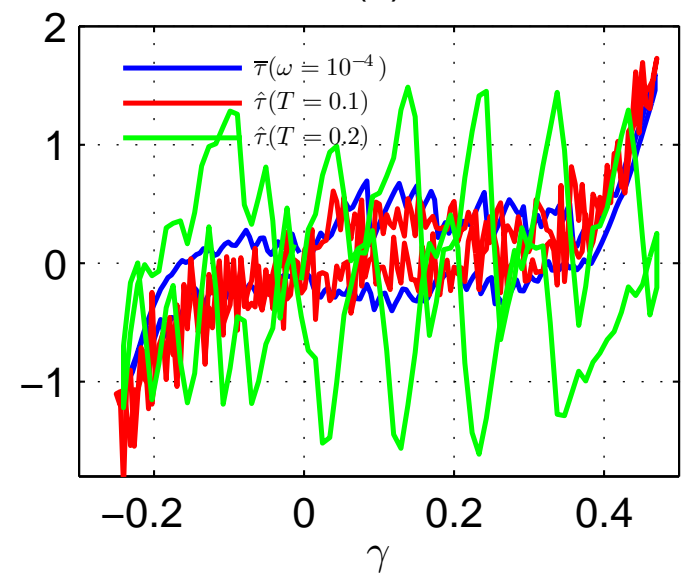

Figure 11: (a) Comparison of the stress-strain curve from fine evolution and PTA. In PTA, the initial particle positions are defined as the last configuration from approximating the Young measures at previous coarse time step while the initial particle velocities are set to be zero at every coarse time step; (b) Comparison of the stress-strain curve from fine evolution and PTA. In the red curve, the initial values (including particle positions and velocities) are chosen to be the last points from the approximation of Young measures at previous coarse time step; In the green curve, the initial positions are chosen to be the first moments of the position function with respect to the approximate Young measures at previous coarse time step while the velocities are set to be zero initially at every step.

Similarly, the overbars are removed for simplicity. We test a system with $L=12 a_{s}, a=5 a_{s}$ and the total number of atoms is 1513. In the initial atomic configuration, we set $a_{s}=1.05 \sqrt{2}$ to make sure that the distance between two nearest neighbors is close to the equilibrium state (i.e. $d^{\mathrm{NiNi}}=1.12246$ ). Then we apply energy minimization to further assure that the atomic system is in (or near) equilibrium. In this case we do NOT employ a thermostat either. Linear increasing displacement is applied at the left and right ends at the same time with opposite signs. We define the strain $\varepsilon$ and the overall stress $\sigma$ as

$$
\begin{aligned}
\varepsilon & =\frac{p_{\text {right }}-p_{\text {left }}}{L}-1.0, \\
\sigma & =\frac{T_{\text {right }}-T_{\text {left }}}{2 \times a \times a},
\end{aligned}
$$

where $p_{\text {left }}$ and $p_{\text {right }}$ denote the averaged atom positions on the left and right sides, respectively. $T_{\text {left }}$ and $T_{\text {right }}$ denote the tensile forces on the left and right sides, respectively.

We first obtain the stress-strain curves from simulating fine-scale dynamics under various strain rates. The results are shown in Figure 14. Oscillations can be observed in high strain rate along the deformation due to inertia effect. With the decrease of the strain rate, the oscillations are smoothed out and the maximum stress is decreased (which is also supported in [8] theoretically and experimentally). Despite of the differences, the constitutive relations in the elastic part are almost the same. Figure 15 presents the stress-strain curve in the case of $\omega=10^{-5}$ with the points $a$ to $j$ on the curve corresponding to the snapshots in $x-z$ plane at different stages shown in Figure 16. Once after energy minimization, the bar is under slight compression due to the internal forces prior to the application of tension, as can be seen in Figure 15 and Figure 16(a). The bar deforms uniformly and elastically with the increasing of the tensile strain before attaining its maximum tensile strength (Figure 16(a)-(d)). Although there is a certain amount of stretch in the horizontal direction, the atoms are organized regularly without any change in its original microstructure ordering. This is followed by a drop in the stress to zero, simply implying the breaking of the atomic bonds. Considerable disorder in 


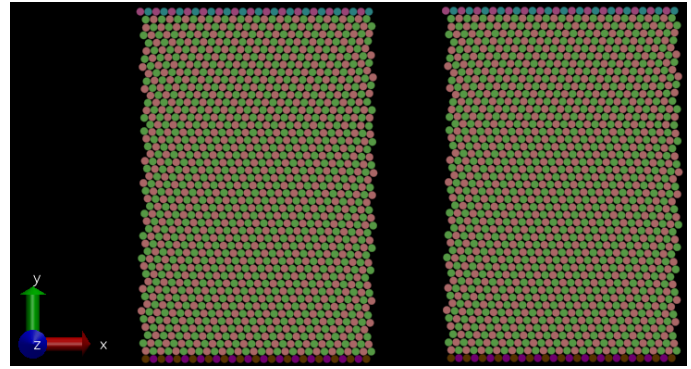

(a) Snapshots at $\mathrm{s}=0$

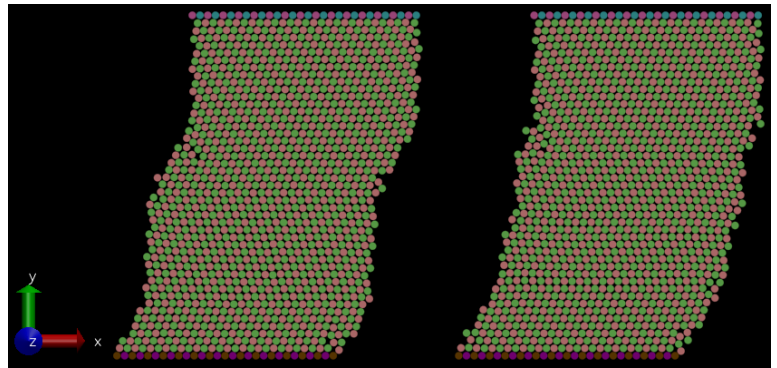

(b) Snapshots at $\mathrm{s}=5$

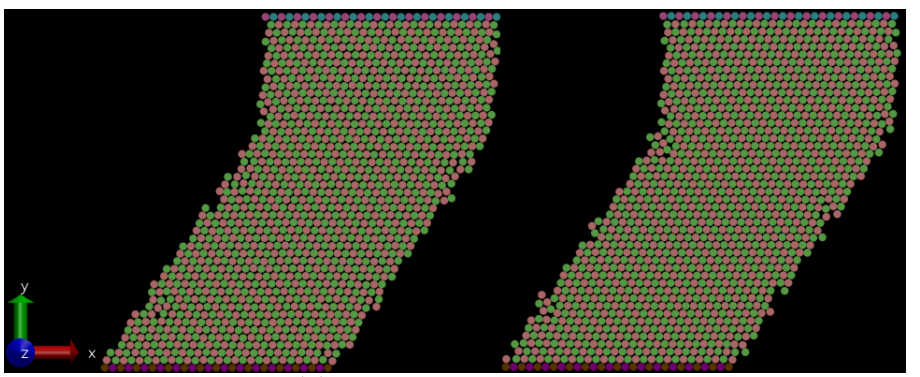

(c) Snapshots at $\mathrm{s}=10$

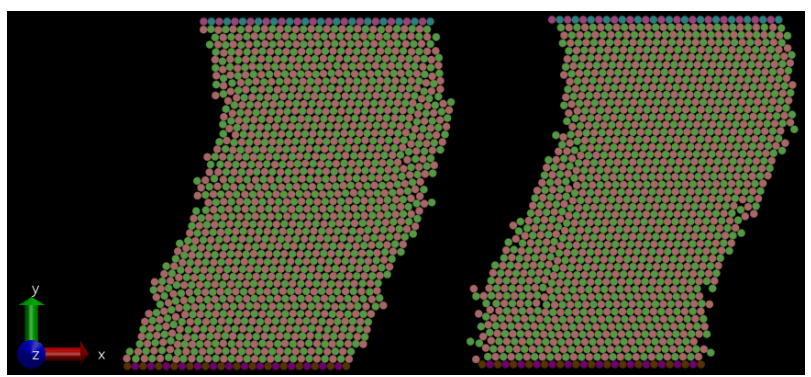

(d) Snapshots at $\mathrm{s}=15$

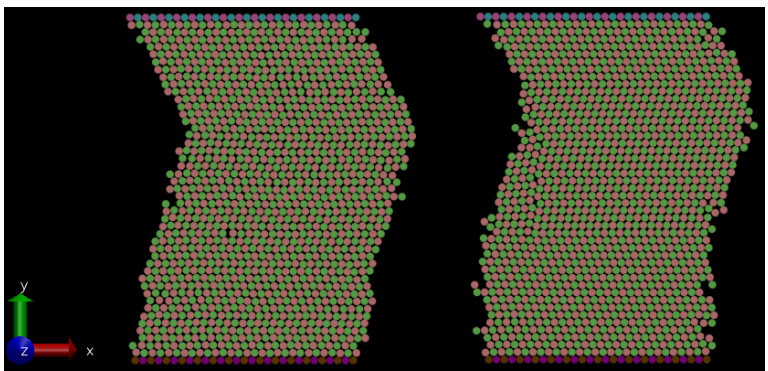

(e) Snapshots at $\mathrm{s}=20$

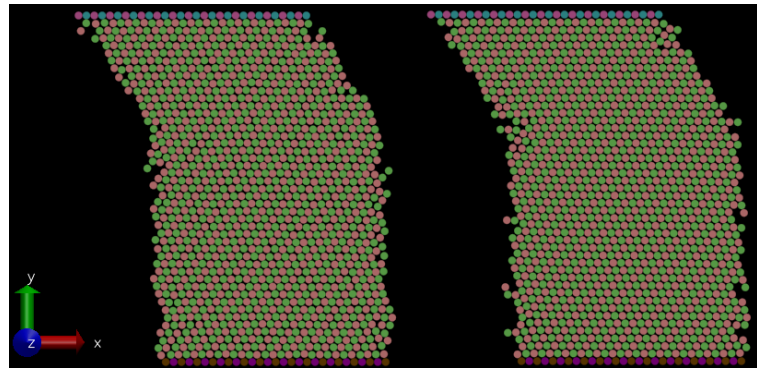

(f) Snapshots at $\mathrm{s}=25$

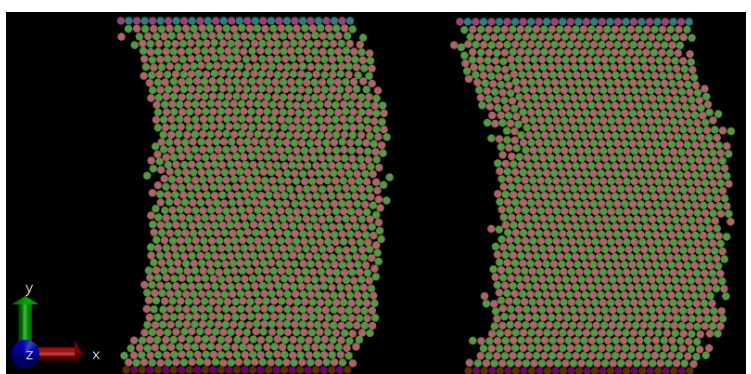

(g) Snapshots at $\mathrm{s}=30$

Figure 12: Snapshots from fine and PTA. In all the figures, the left one shows the snapshots from fine dynamics and the right one shows the snapshots from PTA, which present the approximated Young measures of atom positions rather than the actual configuration from MD. 


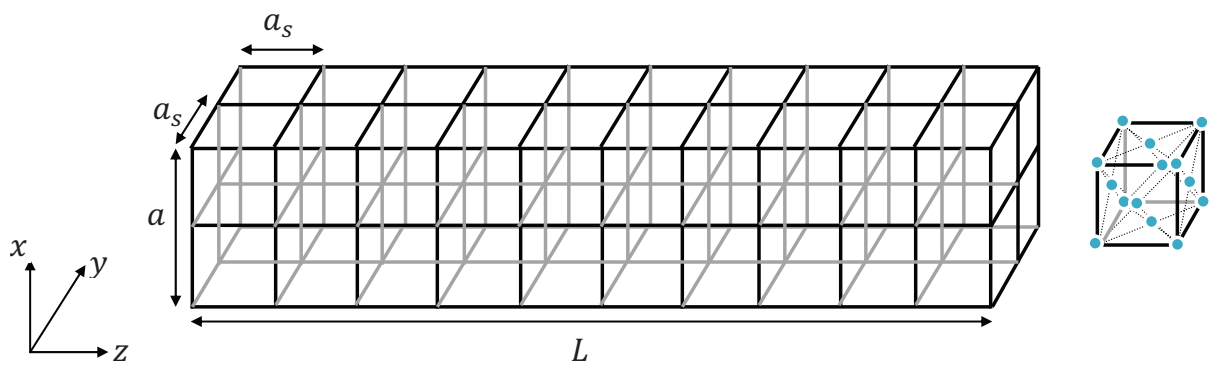

Figure 13: Graphic Illustration of the initial configuration of the 3D Atomic System.

the middle of the bar is observed during this fracture process (Figure 16(e)), with the disorder extending throughout the entire length of the body on subsequent pulling (Figure 16(f)-(i)). A disordered necking region of decreasing diameter can be seen in Figure 16(j) towards the later stage, indicating the total failure of the structure.

We notice that the bar fractures in the uniaxial tension testing as opposed to showing slip/plasticity, probably because of the very few atoms in the $x$ section. This finding is also pointed out in [11]. However, in their case, significant plastic deformation were observed far before the reach of maximum stress, due to dislocation pileup and subsequent rapid motion of dislocations.

We also implement the idea of PTA to obtain the coarse response as $\omega$ tends to zero. Figure 17 shows the results with different coarse time steps. The stress-strain curves in the elastic part match very well but the peaks vary with the coarse time step $T$. We compare the stress-strain relations from fine dynamics and from PTA, and plot a set of consistent results in Figure 18. Following (66) to estimate the time costs, we find $n_{f} / n_{s}=5$ and 50 in the cases of $\omega=10^{-5}$ and $\omega=10^{-6}$, respectively.

\section{Final Remarks}

We summarize the main findings of this paper in this section.

- We find high frequency oscillations of the atoms (thus high kinetic energy) in the atomic chain system. This is due to the inner instability of the material governed by non-convex interaction potentials. The inner vibration of the system is independent of loading rates and

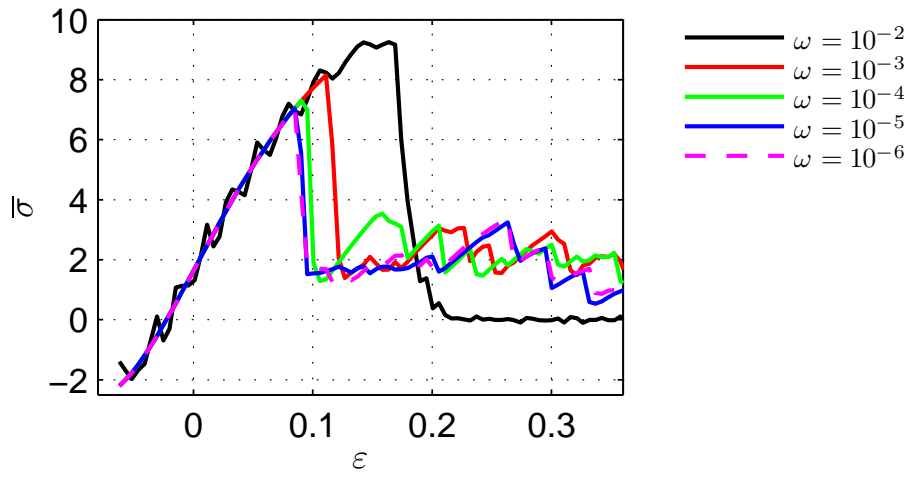

Figure 14: Results from fine dynamics under different loading rates. The black, red, green, blue and magenta curves present the results with loading frequency $\omega=10^{-2}, 10^{-3}, 10^{-4}, 10^{-5}$ and $10^{-6}$ respectively. 


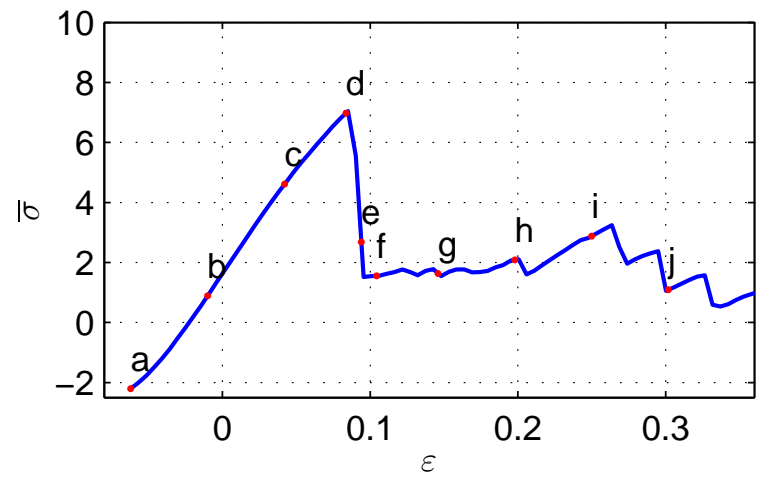

Figure 15: Stress-strain curve from fine dynamics in the case of $\omega=10^{-5}$. The points $a$ to $j$ correspond to the snapshots in Figure 16.

can only be stabilized by applying viscosity or thermostat in the system, which in turn, lead to strong hysteresis. When the loading rate tends to zero, the hysteresis converges to the quasi-static result.

- An interesting observation from the fine-scale dynamics is that in the one-dimensional atomic chain system, the chain becomes unstable beyond the critical strain and undergoes high frequency vibrations while in the case of the two-dimensional Ni-Mn system, the kinetic energy of the 2D lattice is kept low even without any constrain on the temperature of the system. This phenomenon can be explained by different mechanisms of phase transformations. In the first case the atomic chain needs to move from a low-strain equilibrium state to a high-strain equilibrium state where the unstable region is in between (the critical points are denoted by green dots in Figure 19(a)). In the second case, the lattice transforms between two different variants of martensite. The routes between energy minimum points are illustrated in red in Figure 19(a) and (b), respectively. Taking the non-dimensionalized data in (59) and computing $\phi^{\mathrm{NiNi}}$ using (57), we find the red curve in Figure 19(b) is defined as

$$
\left|x_{\beta}-x_{\alpha}\right|^{2}+\left|y_{\beta}-y_{\alpha}\right|^{2}=2^{1 / 3} .
$$

The two variants of martensite correspond to two points on this curve and also satisfy the kinematical compatibility condition. We assure that the lattice deforms within a reasonable range near the equilibrium states, thus keep the deformation away from the instability points, which are located on the green curve.

- PTA describes the limit dynamics of an induced slow-fast system with explicit separation of time scales between the fast and slow motion. Therefore, in the implementation of PTA on MD systems, the assumption of slow loading is essential. This is the limitation of PTA, but this limitation does not influence its application on a class of engineering problems where running the MD systems under slow loading rate is more challenging.

- Our definition of coarse variables as time averages of phase functions provides a systematic procedure for the Young measure theory to be applied. If the coarse variables are chosen to be any arbitrary instantaneous functions of the fine variables, the coarse evolution equation will normally take a singularly perturbed form.

- The Young measures involved here are not unique and can be dependent on fine i.c.s. The limit dynamics in the cases of unique and non-unique Young measures is given in [5], where in the latter case, the limit of the slow motion solves a differential inclusion. In practice we generate the approximate Young measures in a coarse time step by using as initial conditions 


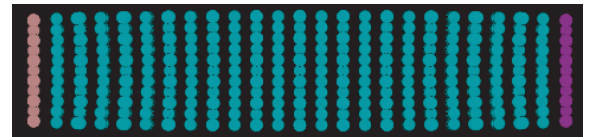

(a) Snapshot at $\mathrm{s}=0$

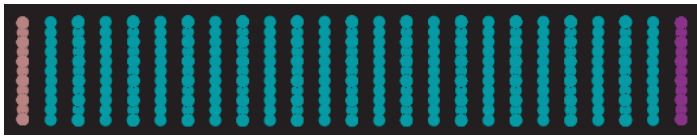

(c) Snapshot at $\mathrm{s}=1$

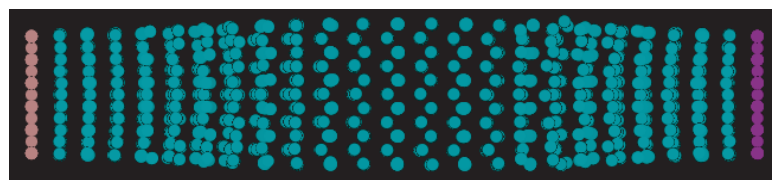

(e) Snapshot at $\mathrm{s}=1.5$

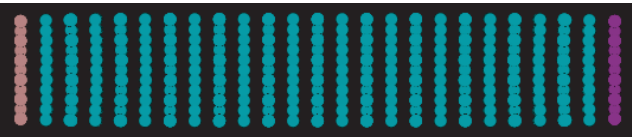

(b) Snapshot at $\mathrm{s}=0.5$

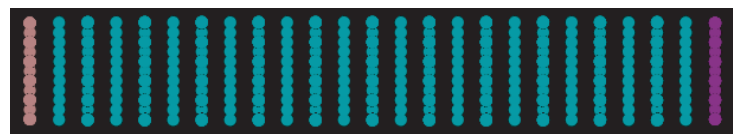

(d) Snapshot at $\mathrm{s}=1.4$

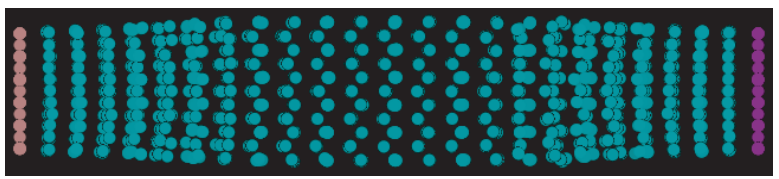

(f) Snapshot at $\mathrm{s}=1.6$

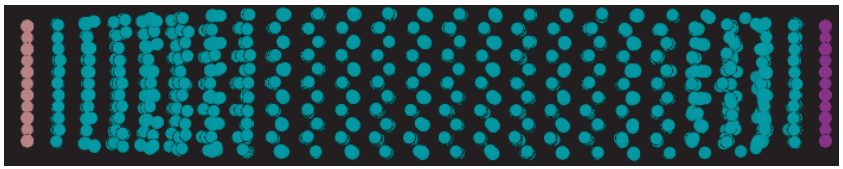

(g) Snapshot at $\mathrm{s}=2.0$

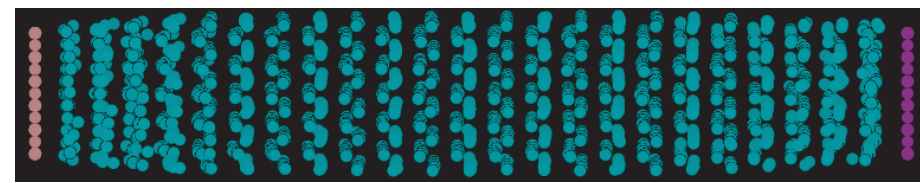

(h) Snapshot at $\mathrm{s}=2.5$

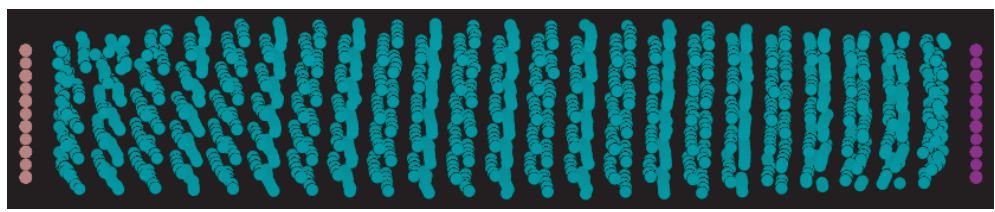

(i) Snapshot at $\mathrm{s}=3$

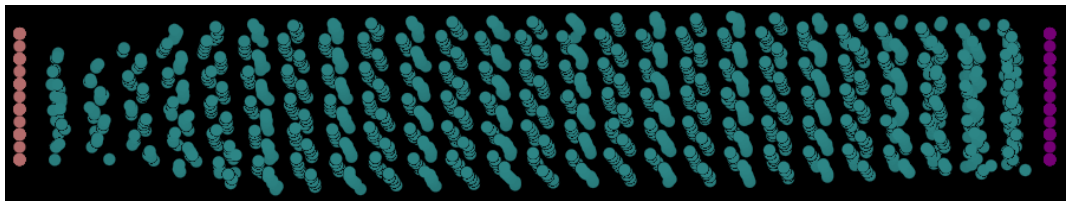

(j) Snapshot at $\mathrm{s}=3.5$

Figure 16: Snapshots from fine dynamics in the case of $\omega=10^{-5}$. Here $s(=\omega t)$ is the slow time which will be used in the implementation of PTA later. 


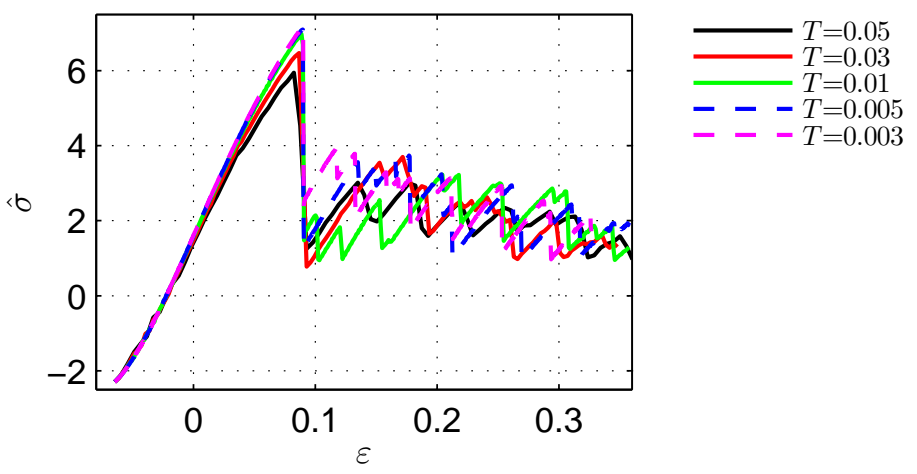

Figure 17: Results from PTA with different coarse time steps. The black, red, green, blue and magenta curves present the results with coarse time steps $T=0.05,0.03,0.01,0.005$ and 0.003 respectively. In P$\mathrm{TA}$, the initial particle positions and velocities are set to be the same as the last point from approximating the Young measures at previous coarse time step.

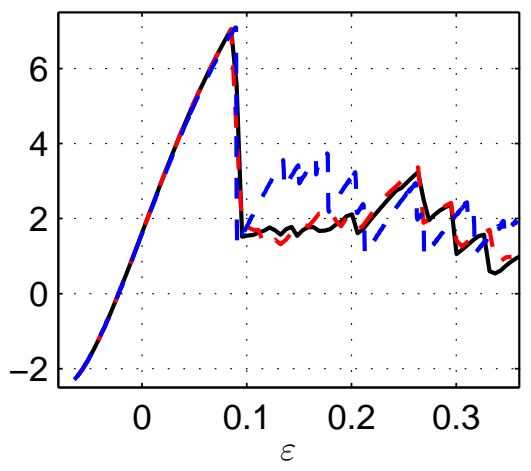

$-\bar{\sigma}\left(\omega=10^{-5}\right)$
$--\bar{\sigma}\left(\omega=10^{-6}\right)$
$--\hat{\sigma}(T=0.005)$

Figure 18: Comparison of results from fine evolution and from PTA. The black and red curves present the results from fine evolution with loading frequencies $\omega=10^{-5}$ and $10^{-6}$ respectively. The blue curve shows the result from PTA with coarse time step $T=0.005$.

(a)

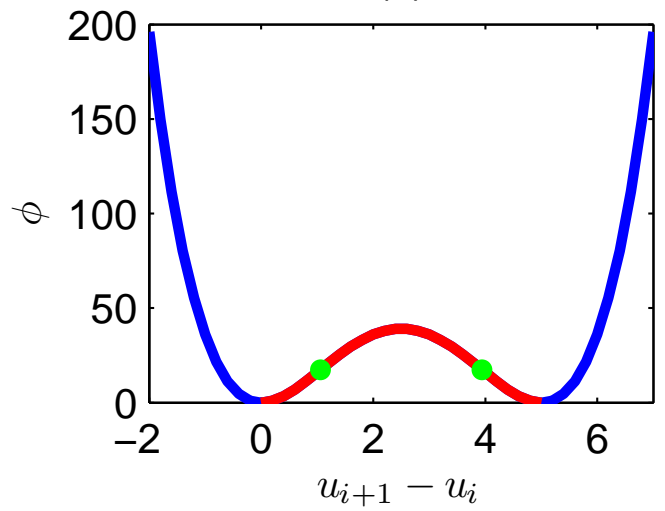

(b)

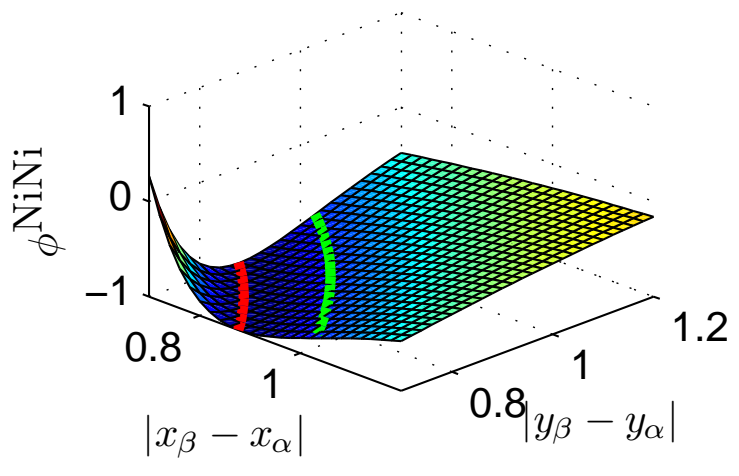

Figure 19: Interatomic potential functions. The red curves indicate the possible energy routes between the minimums of energy. (a) Double-well energy function that is used to describe the interatomic potential in the one-dimensional atomic chain system; (b) Lennard-Jones energy function that is used to describe the interatomic potential in the two-dimensional Ni-Mn system. 
for a fast run the last state from the approximation of the Young measure at the previous coarse step. This strategy seems to work fine in the current (non-trivial) problems, but we have to emphasize that this is only one option, and more sophisticated and systematic methods are required to tackle this issue.

- An essential, required improvement beyond this first study to evaluate the efficacy of PTA is the development of error-estimates for the scheme. In particular, knowledge of upper bounds on coarse time step size would be desirable.

- From the numerical study, we find that at high strain rate in fine-scale dynamics, PTA usually fails to generate consistent coarse response even when the coarse time step is chosen to be small. However, when the strain rate is small, we observe consistent results from PTA. This observation implies a favorable trend since in the range of extremely slow loading, current MD takes unrealistically long simulation time. Thus, PTA could be a good alternative to MD.

- Another finding is that some of the coarse responses from fine are non-smooth. With the implementation of PTA, we successfully predict these non-smooth behaviors. Note that we are essentially solving (55) instead of the system of ODE (48), which relaxes the smoothness requirement of the coarse response.

- The PTA technique was first proposed in [16] and tested there on two model problems. These problems were designed to probe its capability on the most important qualitative feature required of it, that of averaging fast, nonlinear, oscillatory dynamics subject to slowly evolving forcing. Of necessity, these first test problems had to be 'simple' and lowdimensional. In this paper, we focus on the application of PTA on atomistic simulations, giving its first demonstration on realistic models for the mechanics of materials. However, this is not a trivial application - it requires real advances of the methodology, for instance:

- Incorporating our multiscale technique into MD software LAMMPS.

- Defining physically interesting variables and retrieving the associated dynamics at slow time scale.

- Setting appropriate coarse time steps by evaluating accuracy and efficiency of our method compared to direct MD simulation.

- Tackling the problem of non-uniqueness mapping from slow time-scale dynamics to fast MD.

- Exploring the range of strain rate where our method works fine.

We discuss all these issues in the paper and hopefully, our work could shed light on the longstanding open problem of $\mathrm{MD}$, that is, slow process as strain rate goes to zero.

Acknowledgement. The work was partially supported by Civil and Environmental Engineering Department at Carnegie Mellon University through the Ellegood Strategic Doctoral Fellowship and partially supported by NSF (Dynamical Systems, Grant \# 0926579).

\section{References}

[1] Rohan Abeyaratne and James K Knowles. Evolution of phase transitions: a continuum theory, volume 10. Cambridge University Press, 2006.

[2] A. Acharya. On the choice of coarse variables for dynamics. International Journal for Multiscale Computational Engineering, 5(6):483-489, 2007. 
[3] A. Acharya. Coarse-graining autonomous ODE systems by inducing a separation of scales: practical strategies and mathematical questions. Mathematics and Mechanics of Solids, 15(3):342-352, 2010.

[4] Z. Artstein, I.G. Kevrekidis, M. Slemrod, and E.S. Titi. Slow observables of singularly perturbed differential equations. Nonlinearity, 20:2463, 2007.

[5] Z. Artstein and A. Vigodner. Singularly perturbed ordinary differential equations with dynamic limits. In Proceedings of the Royal Society of Edinburgh-A-Mathematics, volume 126, pages 541-570. Cambridge Univ Press, 1996.

[6] Zvi Artstein, C William Gear, Ioannis G Kevrekidis, Marshall Slemrod, and Edriss S Titi. Analysis and computation of a discrete kdv-burgers type equation with fast dispersion and slow diffusion. SIAM Journal on Numerical Analysis, 49(5):2124-2143, 2011.

[7] A.M. Balk, A.V. Cherkaev, and L.I. Slepyan. Dynamics of chains with non-monotone stress-strain relations. i. model and numerical experiments. Journal of the Mechanics and Physics of Solids, 49(1):131-148, 2001.

[8] Richard W Hertzberg, Richard Paul Vinci, and Jason L Hertzberg. Deformation and fracture mechanics of engineering materials, volume 89. Wiley New York, 1996.

[9] F.E. Hildebrand and R. Abeyaratne. An atomistic investigation of the kinetics of detwinning. Journal of the Mechanics and Physics of Solids, 56(4):1296-1319, 2008.

[10] P.H. Hünenberger. Thermostat algorithms for molecular dynamics simulations. Advanced Computer Simulation, pages 130-130, 2005.

[11] R Komanduri, N Chandrasekaran, and LM Raff. Molecular dynamics (md) simulation of uniaxial tension of some single-crystal cubic metals at nanolevel. International Journal of Mechanical Sciences, 43(10):2237-2260, 2001.

[12] Chang-Tsan Lu and Kaushik Dayal. Linear instability signals the initiation of motion of a twin plane under load. Philosophical Magazine Letters, 91(4):264-271, 2011.

[13] Steve Plimpton et al. Fast parallel algorithms for short-range molecular dynamics. Journal of Computational Physics, 117(1):1-19, 1995.

[14] P.K. Purohit. Dynamics of phase transitions in strings, beams and atomic chains. 2002.

[15] Marshall Slemrod and Amit Acharya. Time-averaged coarse variables for multi-scale dynamics. Quarterly of applied mathematics, 70(4):793-803, 2012.

[16] Likun Tan, Amit Acharya, and Kaushik Dayal. Coarse variables of autonomous ode systems and their evolution. Computer Methods in Applied Mechanics and Engineering, 253:199$218,2013$.

[17] LV Woodcock. Isothermal molecular dynamics calculations for liquid salts. Chemical Physics Letters, 10(3):257-261, 1971.

[18] Yubao Zhen and Anna Vainchtein. Dynamics of steps along a martensitic phase boundary ii: Numerical simulations. Journal of the Mechanics and Physics of Solids, 56(2):521-541, 2008 . 